\title{
Probing surfaces and interfaces in complex oxide films via in situ X-ray photoelectron spectroscopy
}

\author{
Suresh Thapa ${ }^{\mathrm{b}}$, Rajendra Paudel ${ }^{\mathrm{b}}$, Miles D. Blanchet ${ }^{\mathrm{b}}$, Patrick T. Gemperline ${ }^{\mathrm{b}}$, Ryan B. Comes ${ }^{\mathrm{a})}$
} (10)

Department of Physics, Auburn University, Auburn, Alabama 36849, USA

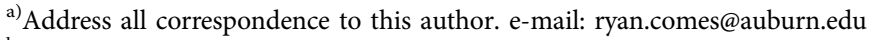

${ }^{\mathrm{b}}$ These authors contributed equally to this work.

Received: 1 June 2020; accepted: 27 August 2020; published online: 4 February 2021

Emergent behavior at oxide interfaces has driven research in complex oxide films for the past $\mathbf{2 0}$ years. Interfaces have been engineered for applications in spintronics, topological quantum computing, and high-speed electronics with properties not observed in bulk materials. Advances in synthesis have made the growth of these interfaces possible, while X-ray photoelectron spectroscopy (XPS) studies have often explained the observed interfacial phenomena. This review discusses leading recent research, focusing on key results and the XPS studies that enabled them. We describe how the in situ integration of synthesis and spectroscopy improves the growth process and accelerates scientific discovery. Specific techniques include determination of interfacial intermixing, valence band alignment, and interfacial charge transfer. A recurring theme is the role that atmospheric exposure plays on material properties, which we highlight in several material systems. We demonstrate how synchrotron studies have answered questions that are impossible in lab-based systems and how to improve such experiments in the future.

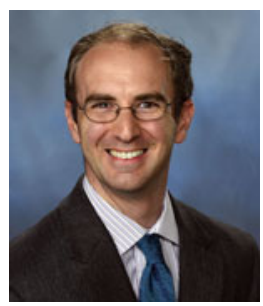

Dr. Ryan Comes is an experimental condensed matter and materials physicist and has been an assistant professor of physics at Auburn University since 2016. At Auburn, he is the principal investigator of the Films, Interfaces, and Nanostructures of Oxides (FINO) Lab. The group's research focuses on the synthesis and characterization of complex oxide thin films and nanostructures through molecular beam epitaxy deposition and in situ X-ray photoelectron spectroscopy. Prior to joining Auburn, he was the Linus Pauling Distinguished Postdoctoral fellow at Pacific Northwest National Laboratory from 2013 to 2016. He received his B.S. in Physics and Electrical and Computer Engineering from Carnegie Mellon University and Ph.D. in Engineering Physics from the University of Virginia in 2013. He has received various awards for his research, including a 2020 Air Force Young Investigator Award, the Materials Research Society Graduate Student Silver Award in 2016, and the National Defense Science and Engineering Graduate Fellowship in 2010. His teaching at Auburn has also been recognized in the form of the Society of Physics Students' Most Outstanding Professor Award in 2020.

\section{Introduction}

From multiferroics to two-dimensional electron gases (2DEGs) to strongly correlated and topological systems, much of complex oxide thin film research has been driven by interfacial phenomena for the past 20 years. Groups have demonstrated emergent oxide 2DEGs-also referred to as $2 \mathrm{D}$ electron systems and 2D electron liquids-due to polar/non-polar interfaces [1], multiferroic behavior with magnetoelectric control from interfaces between oxide ferroelectrics and ferromagnets
[2, 3], and emergent ferromagnetism [4] as well as orbital polarization [5] due to interfacial charge transfer. Numerous groups have reviewed the novel properties that have been predicted and reported at oxide interfaces, and readers are referred to several recent review articles summarizing the state-of-the-art research in oxide thin films $[6,7,8]$. Advances in thin film synthesis continue to drive the field as well. New approaches to molecular beam epitaxy (MBE) [9, $10,11]$ and pulsed laser deposition (PLD) $[12,13]$ enable 
more precise control of cation stoichiometry than previously possible. Such approaches are highly complementary with materials characterization techniques that help to explain the physical origins of emergent phenomena in oxide thin films and interfaces.

In this review, we will discuss how the reported interfacial phenomena can be examined using surface science tools-particularly in situ X-ray photoelectron spectroscopy (XPS)-in concert with film synthesis and other ex situ techniques to best understand the properties of oxide thin films, surfaces, and interfaces. This is illustrated conceptually in Fig. 1, with synthesis and spectroscopy integrated in a single vacuum system to produce a feedback loop for the study of films and interfaces. Our goal here is to introduce XPS to a synthesis-focused audience by showing how the technique can be applied to improve film growth and accelerate experimental studies of interfacial phenomena. We also demonstrate the importance of considering post-growth spectroscopy measurements during the experimental planning process, which is vital to the success of both in situ and ex situ spectroscopy measurements. Finally, a recurring theme throughout this review is that researchers must be aware of chemical effects that occur on the film surface after growth, beginning with sample cooldown all the way to atmospheric exposure and subsequent ex situ measurements. These chemical changes are often observable in XPS measurements, but it is easy to ignore their impacts on other experimental results.

Beginning with several recent examples from the literature that motivate the importance of in situ characterization, we present a brief overview of the physics of XPS and how to design experiments that best integrate thin film synthesis and in situ XPS to achieve high-quality thin films and high impact scientific results. We then present several examples of how XPS has been used in concert with film synthesis to better understand surface properties, interfacial charge transfer, electronic band alignment across a heterojunction, and non-idealities such as intermixing and off-stoichiometry. Finally, we present an overview of future opportunities that can leverage user facilities and the lessons learned from lab-based in situ studies for further breakthroughs.

\section{Overview of complex oxide thin films and interfaces}

The pursuit of a high-mobility 2DEG at complex oxide interfaces has driven research in a wide variety of materials for more than a decade. Such 2DEGs offer the promise of higher carrier concentrations than can be achieved with other materials systems, making them exciting candidates for high-speed electronics. Oxide 2DEGs also offer additional benefits over their semiconductor counterparts in that they can exhibit

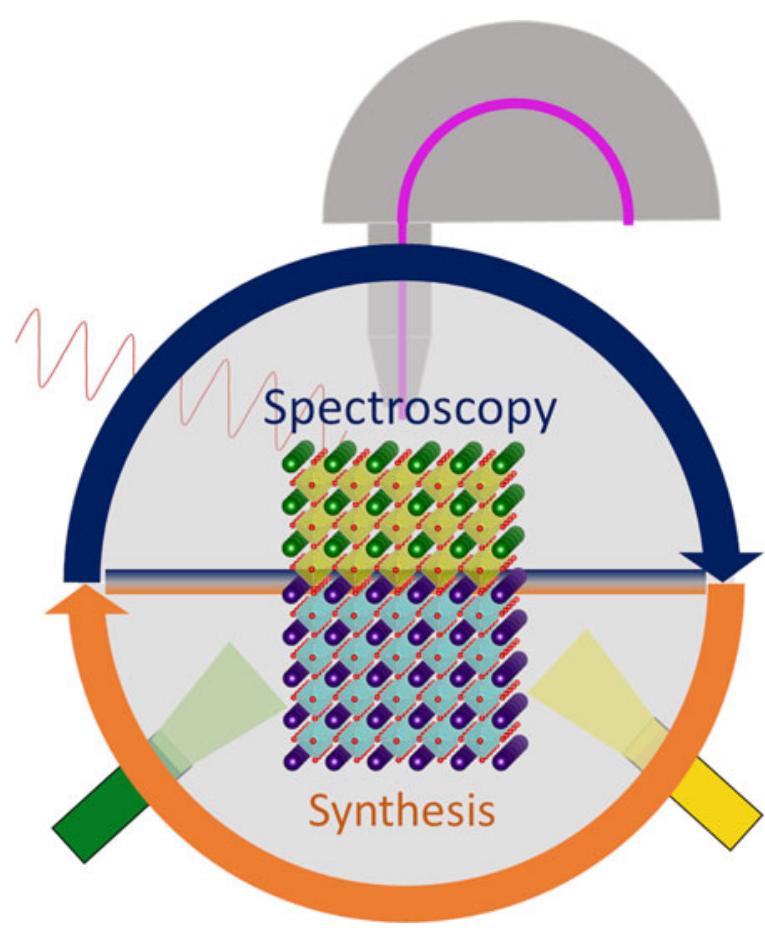

Figure 1: Feedback loop of film synthesis and in situ XPS for the study of oxide thin films and interfaces, highlighting how interfacing in situ X-ray spectroscopy capabilities can feedback to improve the synthesis process while also providing critical information about interfacial phenomena.

strong spin-orbit coupling when comprised of $5 d$ electrons, such as those in $\mathrm{KTaO}_{3}$ [14]. Spin-orbit coupling in a 2DEG offers an additional opportunity not available in conventional semiconductor devices. Beginning with the $\mathrm{LaAlO}_{3} / \mathrm{SrTiO}_{3}$ interface [1], many candidate materials systems have been explored. The $\mathrm{LaAlO}_{3} / \mathrm{SrTiO}_{3}$ system was proposed for transistors with high mobility and high carrier concentrations [15, 16], spin-orbit coupled heterostructures with Rashba splitting $[17,18]$, and numerous other applications. However, controversy regarding the role of defects in the $\mathrm{LaAlO}_{3} / \mathrm{SrTiO}_{3}$ system $[19,20,21]$ has been present for many years. As we will discuss later, XPS along with a variety of other complementary characterization techniques helped to explain the physical origin of the $2 \mathrm{DEG}$ in these materials $[19,22,23]$.

Subsequent materials research has focused on alternative structures, including interfaces between $\mathrm{SrTiO}_{3}$ and rare-earth titanates containing a $\mathrm{Ti}^{3+}$ cation, such as in $\mathrm{NdTiO}_{3} / \mathrm{SrTiO}_{3}$ [24, 25], $\mathrm{GdTiO}_{3} / \mathrm{SrTiO}_{3}$ [26], and $\mathrm{LaTiO}_{3} / \mathrm{SrTiO}_{3}[27,28]$. Here, delta-doping of electrons produces the 2DEG, with the rare-earth A-site ion providing an additional electron for the system. Emergent phenomena have also been reported in these interfacial materials, including superconductivity [28] and ferromagnetism $[29,30]$. As with the $\mathrm{LaAlO}_{3} / \mathrm{SrTiO}_{3}$ system, however, defects can still play a significant role, albeit in a different fashion. Various groups have reported excess oxygen content in rare-earth titanates $[25,31,32]$ due to either 
imperfect tuning of oxygen composition during growth or atmospheric exposure after growth, which has been confirmed through ex situ XPS in each case. These changes in oxygen stoichiometry can have profound impacts on material properties, changing conductivity [25], and magnetic response [32]. When such properties are observed, it is often difficult to decouple surface chemistry and defects from emergent physical phenomena, making in situ spectroscopy valuable.

With the emerging emphasis on materials for quantum computation, materials that exhibit strong spin-orbit coupling have generated an increased interest in the oxide community as well. Work has focused on $5 d$ transition metal oxides materials such as $\mathrm{KTaO}_{3}[14,33]$, which exhibits the Rashba effect, and $\mathrm{SrIrO}_{3}$, which possesses stronger spin-orbit coupling. In the case of $\mathrm{KTaO}_{3}$, surface 2DEGs have been reported in ultrahigh vacuum conditions from oxygen vacancies [33, 34]. Since these reports, a variety of means have been developed to produce interfacial 2DEGs in $\mathrm{KTaO}_{3}$, primarily through the introduction of polar/non-polar interfaces [35, 36, 37]. These interfaces have exhibited such interesting properties as optically tunable Rashba effect and greater Hall mobility than analogous $\mathrm{LaAlO}_{3} / \mathrm{SrTiO}_{3}$ interfaces. Greater Hall mobility is due to the wider bandwidth of $5 d$ electrons than $3 d$ electrons [36]. As in previous examples, however, a more complete understanding of $\mathrm{KTaO}_{3}$ properties was gleaned from an in situ-cleaved single crystal through a combination of scanning tunneling microscopy (STM), ion scattering spectroscopy, and XPS [38]. In this work, the authors showed that to compensate for the polar $\mathrm{KTaO}_{3}$ surface, a series of physical and chemical distortions occur and that the most stable surface is passivated by water adsorption, as one would expect in samples exposed to atmosphere.

Perovskite iridates are another class of $5 d$ oxides that have generated excitement for their potential in materials systems by taking advantage of topological phenomena $[39,40] . \mathrm{SrIrO}_{3}$ is a semimetallic oxide that exhibits strong spin-orbit coupling with evidence of a dimensionality driven cross-over between metallic and semiconducting behavior in ultra-thin films [41, 42]. In oxide heterostructures, $\mathrm{SrIrO}_{3}$ interfaces have been studied extensively for magnetic and electronic phenomena that occur due to charge transfer and electron correlations. In $\mathrm{STO} / \mathrm{SrIrO}_{3}$ superlattices, others have shown the emergence of an energy gap at the Fermi level as the thickness of the iridate layers is reduced to $\sim 4$ u.c. [43]. They also observed the formation of electronic energy features resembling a Dirac-cone, suggesting that topological effects occur in the ultra-thin limit. (111)-oriented $\mathrm{SrIrO}_{3} / \mathrm{SrTiO}_{3}$ heterostructures have also been synthesized and proposed as topological materials that exhibit the desirable honeycomb lattice for topological quantum information systems [44]. Similar (100)-oriented $\mathrm{SrIrO}_{3} / \mathrm{SrTiO}_{3}$ superlattices have also been shown to behave like layered Ruddelsden-Popper iridates by reducing the iridate layer to 2 unit cells (UCs) [45]. The $\mathrm{SrMnO}_{3} / \mathrm{SrIrO}_{3}$ interface has also been studied through superlattice synthesis and shown to exhibit ferromagnetism due to electron transfer from Ir to $\mathrm{Mn}$ across the interface, leading to the anomalous Hall effect in these structures $[46,47] . \mathrm{LaMnO}_{3} / \mathrm{SrIrO}_{3}$ interfaces have also demonstrated the Rashba effect, with XPS and $\mathrm{X}$-ray absorption measurements confirming electron transfer from Ir to Mn [48].

Strongly correlated oxides such as nickelates have been a long-standing area of interest within the oxide community, due to their potential to exhibit superconductivity that is analogous to the layered cuprates. Efforts have broadly focused on methods to induce orbital polarization in these materials to create a quasi-two-dimensional plane of nickel ions that resembles the planar cuprate structure. Groups have shown that by heterostructuring $\mathrm{LaNiO}_{3}$ into a superlattice with $\mathrm{LaTiO}_{3}$, orbital polarization can be induced to break the symmetry of the $\mathrm{Ni}$ $e_{\mathrm{g}}$ energy levels $[5,49]$. We will discuss ways to examine this type of charge transfer by in situ XPS later in this article. Nickelate thin films have also been shown to exhibit conductivity that is dependent on film thickness and surface termination $[50,51,52,53,54]$. Theoretical predictions have suggested that this phenomenon is due to structural distortions in the surface layer [51], but recent work employing XPS and X-ray absorption spectroscopy (XAS) has suggested that surface oxygen vacancies could also be the cause [55]. In fact, transport models have explicitly treated the surface of $\mathrm{LaNiO}_{3}$ as having a different conductivity from the bulk of the film [54], which would agree with the model put forward from spectroscopy. This provides a good example of the importance of considering chemical differences between the film surface and bulk when interpreting results of other physical property measurements. Recently, an infinite-layer ( $\mathrm{Nd}, \mathrm{Sr}) \mathrm{NiO}_{2}$ nickelate heterostructure has demonstrated superconductivity for the first time $[56,57,58,59]$. Future studies of superconducting nickelates using X-ray spectroscopy will hopefully provide further insights into the very exciting initial results.

In summary, epitaxial complex oxide thin films continue to be a fruitful area of exploration in the condensed matter physics and fundamental materials science communities. However, the complexity of the perovskite oxide materials system in terms of bulk defect tolerance and non-idealities at film surfaces and interfaces makes careful materials characterization vital to best explain the wide range of emergent properties that have been reported in oxide heterostructures. Our goal in this review is to show how XPS measurements have been used in the past to answer many of these questions such that the reader may better be able to employ the versatile technique in future studies. We also aim to provide further insights and interpretations of the current oxide interface literature, which 
is awash in exciting breakthroughs but also is prone to misinterpretation of XPS and complementary spectroscopy data.

\section{Physics of XPS}

The photoelectric effect is the phenomenon at the core of XPS measurements. It is so called because photons propagating through a material are absorbed by core-level and valence electrons in atoms and the resulting energy of the excited electrons is enough to overcome the electrostatic potential of the material and escape into vacuum. The photoelectric effect was first discovered in 1887 by Heinrich Hertz [60], but it was not until 1905 when Albert Einstein integrated the quantization of the light into the photoelectric effect that it was fully understood [61]. Einstein's discovery that the energy is $E_{\text {photon }}=h v$, where $h$ is Plank's constant and $v$ is the frequency of light, subsequently proved the wave-particle duality of light. Kai Siegbahn later won the Nobel Prize for his invention of XPS (then referred to as "electron spectroscopy for chemical analysis") using hard X-rays generated by a Mo $\mathrm{K}_{\alpha 1}$ and $\mathrm{K}_{\alpha 2}$ source with photon energies of 17.48 and $17.37 \mathrm{keV}$, respectively [62].

Assuming we precisely control the wavelength of light using a Rowland circle monochromator, we may determine the binding energy of an electron via the equation:

$$
E_{\text {photon }}=h v=E_{\mathrm{B}}+\Phi_{\mathrm{S}}+E_{\mathrm{k}^{\prime}}
$$

where the binding energy, $E_{\mathrm{B}}$, is the energy needed to bring it to the Fermi level, the material's work function, $\Phi_{S}$, is the energy needed to free an electron at the Fermi level and get it to vacuum, and the vacuum kinetic energy is $E_{\mathrm{k}}$. The historical three-step model is popular to understand the physics of photoemission qualitatively, with the steps defined as: (a) optical absorption of the incoming X-ray by an atom, (b) electron transport to the surface of the material, and (c) transmission through the surface [63]. We attempt to address this process briefly here and refer to the reader to texts on the physics of XPS for greater detail $[64,65]$.

XPS works by directing an X-ray beam generated by a monochromatic X-ray source onto a sample as shown in Fig. 2. The photoelectric effect causes bound electrons to be ejected out of the sample where they pass through an analyzer. This analyzer uses electron optics to select only electrons with a certain kinetic energy. By varying the settings of the optics, the XPS can sweep a range of energies. Modern XPS systems employ analyzers that allow for several electron energies to be detected simultaneously, thus making data collection more efficient $[66,67,68]$. To connect the photon energy and the kinetic energy of the electrons measured by the XPS, it is best to think about the electronic band alignment diagram of XPS in Fig. 2.

There are techniques to determine the sample's work function, but there is an immediate problem in that the kinetic energy measured by the detector, $E_{\mathrm{K}}$, is not the same kinetic energy of the emitted electrons, $E_{\mathrm{K}^{\prime}}$. Due to biasing of the optics and electron energy analyzer, there is a work function of each XPS, $\Phi_{\mathrm{A}}$, that relates $E_{\mathrm{K}}$ to $E_{\mathrm{K}^{\prime}}$. However, if the sample is mounted on a stage that shares a common ground with the XPS analyzer, then we can equate the Fermi levels of the sample and XPS. Now by looking at the band diagram, we can see that $E_{\mathrm{K}^{\prime}}=E_{\mathrm{K}}+\Phi_{\mathrm{A}}-\Phi_{\mathrm{S}}$. Substituting this into Eq. (1), we get $h v=E_{\mathrm{B}}+E_{\mathrm{K}}+\Phi_{\mathrm{A}}$ or $E_{\mathrm{B}}=h v-E_{\mathrm{K}}-\Phi_{\mathrm{A}}$. So, by using the optics of an XPS to select and count only electrons with $E_{\mathrm{K}}$ and by controlling the X-ray source frequency, we are able to calculate the binding energy of each electron excited by the photoelectric effect without knowing the sample work function, $\Phi_{\mathrm{S}}$. The probability of photoelectrons escaping from the material is also sensitive to the angle of emission from a planar surface and the kinetic energy of the electron. These features are governed by the inelastic mean free path (IMFP), $\lambda$, of an electron within the material [69], which can be modeled by the equation:

$$
\lambda(\mathrm{nm})=\frac{143}{E_{\mathrm{k}^{\prime}}^{2}\left(\mathrm{eV}^{2}\right)}+0.054 * \sqrt{E_{\mathrm{k}^{\prime}}(\mathrm{eV})} .
$$

Figure 3 shows a graph of the IMFP for different X-ray sources (Fig. 3(a)) and different elemental core levels for $\mathrm{Al}$ $\mathrm{K} \alpha$ radiation (Fig. 3(b)). While Eqs. (1) and (2) describe the measurement of electron binding energy via XPS, separate physical phenomena govern the observation of peaks at various binding energies. Of these phenomena, the spin-orbit coupling of core-level electrons, multiplet splitting, and satellite peaks are some of the most commonly observed. However, when it comes to understanding XPS data, these are not always accurately interpreted [70]. Each of these will be covered briefly in this section. Auger electrons are also detected during an XPS measurement and can be beneficial for elemental identification but are also a source of frustration if the kinetic energy of the Auger electron is close to that of an elemental core level (Fig. 3).

Because of the nature of photoelectron scattering within the crystal prior to escape, XPS measurements are inherently surface sensitive. For a given atomic species with volume density $\rho$, the measured intensity as a function of emission angle $\theta$ relative to the surface normal is given by:

$$
I(\theta)=I_{0} \sigma_{\mathrm{n}, 1} T\left(E_{\mathrm{k}}, \theta\right) A \int_{0}^{\infty} \rho(z) e^{-\frac{z}{\cos (\theta)}} \mathrm{d} z,
$$

where $I_{0}$ is the intensity for $\theta=0^{\circ}, \sigma_{n, 1}$ is the absorption cross section for a given energy level with principal quantum number $\mathrm{n}$ and orbital angular momentum $1, A$ is the measured area on the sample, $z$ is the depth within the material, $T\left(E_{\mathrm{k}}, \theta\right)$ is the analyzer transmission function, and $\lambda$ is the IMFP. This equation is generally simplified by assuming that the transmission 

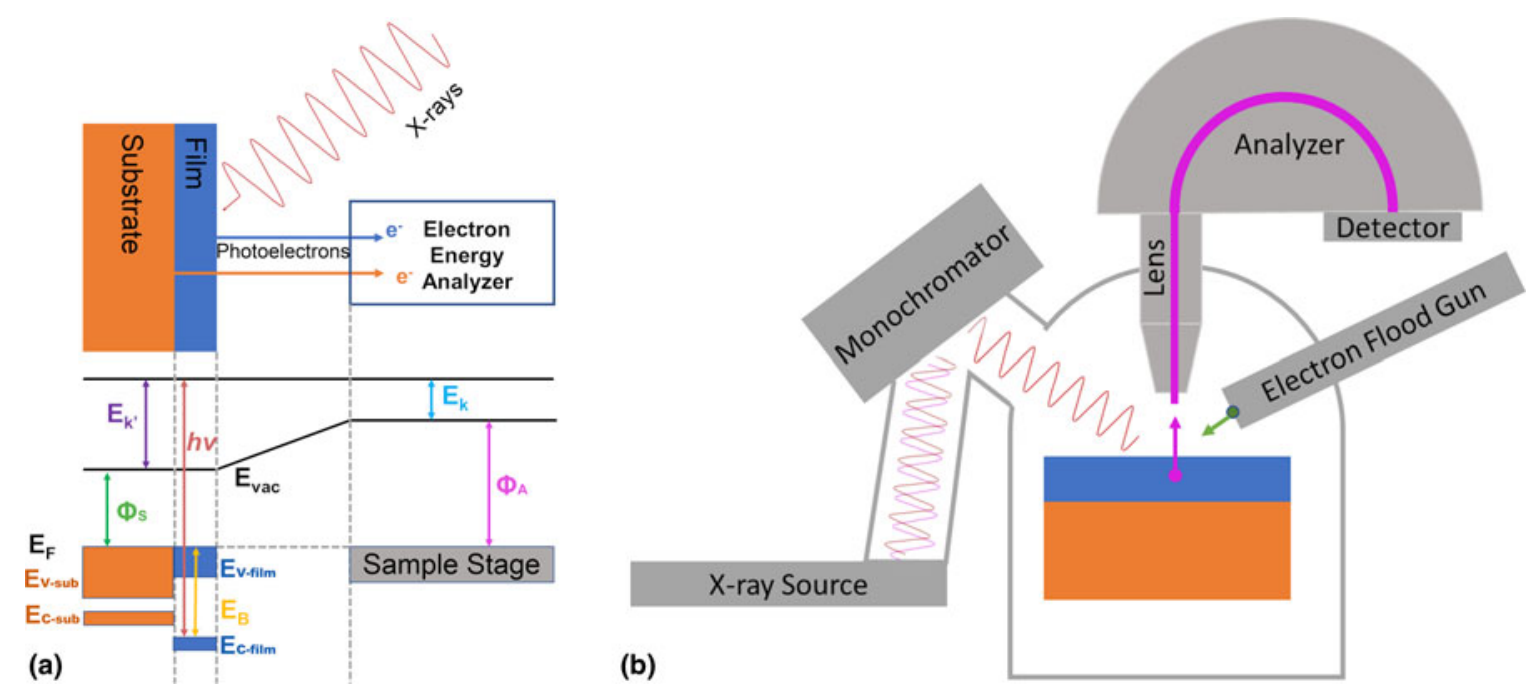

Figure 2: (a) Energy schematic for photoemission from a thin film heterostructure. The sample stage shares a ground with the analyzer causing the Fermi levels of the sample and stage to be equal. The photoelectrons are excited by the incoming X-rays and causing them to escape from the valence and core levels of the substrate and film (left). They are collected by the electron energy analyzer (right) and the binding energy is calculated. (b) Schematic of XPS vacuum chamber configuration, showing non-monochromatic X-ray source, Rowland circle monochromator, hemispherical electron energy analyzer with lens and detector, and neutralizing electron flood gun.

function is constant over the energy values of interest and by using ratios between core-level intensities to eliminate the role of $I_{0}$ and $A$. For more detailed descriptions of the physics of depth sensitivity in XPS, the reader is referred to the work of Powell and Tanuma [71]. Angle-resolved XPS measurements make clever use of the angular and depth dependence of the intensity to extract depth profiles for chemical concentrations and electronic states, which we will detail in "Combining Growth and XPS to Measure Film Quality."

The first additional effect to consider is that of spin-orbit coupling, which is referred to as an initial state effect because it is governed by the energetic configuration of the system prior to photon absorption. Electrons with the same non-zero orbital angular momentum quantum number (i.e., $p, d, f$ ) do not all have the same binding energy. This is because spin-up and spin-down electrons have different total angular momenta. The energy shift caused by this spin-orbit coupling goes as $L *$ $S$, where $L$ and $S$ are the orbital and spin angular momentum operators, respectively. Thus, for all orbitals where $l \neq 0$, there will be a splitting of the binding energy for spin-up and spindown electrons. The two peaks correspond to the two possible values of the total angular moment of the photoelectrons, $j=l$ $\pm s$. For a $2 p$ electron, the total angular momentum can take either a value of $j=1+1 / 2=3 / 2$ or $j=1-1 / 2=1 / 2$. This phenomenon can be seen in the splitting of the Co $2 p$ into the two largest peaks of Fig. 4. Additionally, the ratio of the intensities of the peaks are seen not to be 1:1 but is instead 2:1. This is because the relative intensities are determined by the number of magnetic sub-state configurations, $2 j+1$, that correspond to each $j$ value. Knowing the expected branching ratio of the peak intensities is important when fitting XPS spectra as it allows for other effects to be distinguished from the spin-orbit coupling.

Multiplet splitting arises from the coupling of the angular momentum of two electrons in different orbits [72]. If an atom has an unpaired core electron and an unpaired valence electron, the two electrons' momenta can couple and result in multiple peaks. This coupling follows the L-S or RussellSaunders coupling scheme. The peaks are separated based on the total angular momentum, $j$, of the coupled electrons. Spin-orbit coupling depends only on the single photoelectron emitted and thus is consistent and easily predictable effect. In the case of multiplet splitting, however, the splitting depends on both the core electron and valence electrons in the same atom. Additionally, given the presence of multiple unpaired valence electrons, the core electron can pair with any of them and each could yield a different energy shift. Multiplet splitting is commonly observed in transition metal ions that have partially filled $d$ orbitals. For example, the valence of Mn can be determined from the multiplet splitting of the Mn $3 s$ core level [73], while different valences and atomic coordinations of $\mathrm{Cr}$ exhibit differing multiplet features [74]. The variety of final-state coupling energies can make for highly complex spectra with several distinct energy features summing together to produce, for example, a $\mathrm{Cr} 2 p_{3 / 2}$ peak. In general, multiplet splitting will occur for any transition metal ion that has nonzero spin in the $d$ band and care must be taken not to inaccurately attribute multiplet-split peaks to chemical shifts from differing formal charge.

In addition to multiplet splitting, shake-up satellite peaks occur when an atom is photo-ionized, which results in core- 


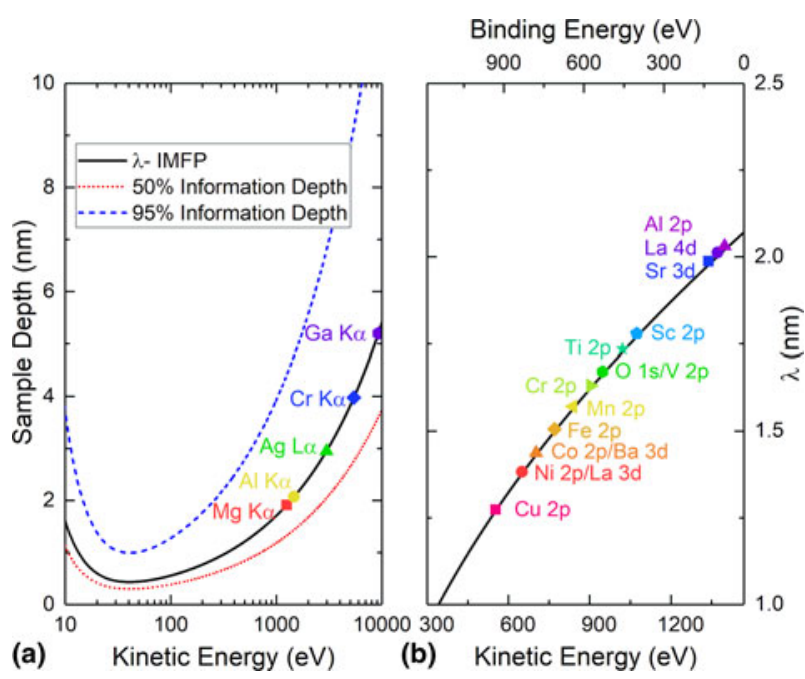

Figure 3: (a) Universal curve of the photoelectron mean free path, $\lambda$, as a function of electron kinetic energy. Dotted and dashed lines show the $50 \%$ (red, dotted) and $95 \%$ (blue, dashed) information depths in the sample for a given kinetic energy. Various lab X-ray source photon energies are labeled on the curve to show the maximum value of $\lambda$ that can be achieved from that source. (b) IMFP for an Al K $\mathrm{K}_{\mathrm{a}}$ (photon energy =1487 eV) source based on Eq. (2) for $3 d$ transition metal $2 p$ peaks as well as relevant peaks for $\mathrm{Al}$, $\mathrm{La}, \mathrm{Ba}$, and $\mathrm{Sr}$.

level hole and subsequent electronic rearrangement [75]. In a shake-up shift, an electron in the valence level transitions to an unoccupied non-degenerate energy level after photoionization alters the electrostatic potential of the atom. This leaves the atom in an excited state that can be modeled using the sudden approximation and simultaneously reduces the kinetic energy of the photoemitted electron. In this way, shake-up is referred to as a final-state effect because the satellite originates from the quantum state formed in the system after the electron has been excited. This reduced kinetic energy of the photoelectron results in a satellite peak that appears to have a greater binding energy than the primary elemental core-level peak. For example, $\mathrm{Co}^{2+}$ ions exhibit a large shake-up satellite peak at higher binding energy [76], as can be seen in Fig. 4. The peak shape and difference in binding energy between the primary peak and the satellite can be a valuable tool for the identification of cation valence, as in the case of Co where the peak shift is fairly small between $\mathrm{Co}^{2+}$ and $\mathrm{Co}^{3+}$ but the satellite structure is markedly different. Furthermore, chemical coordination changes the selection rules for electronic energy level transitions, making shake-up satellites sensitive to ionic coordination as well as formal charge [75].

A related type of final-state satellite peaks come from charge transfer between atoms. When an atom is photoionized, it may result in a change of the electrical structure of itself and an adjacent atom. This change in ionization and electron configuration causes a change in the potential photoelectrons feel and is again measured as a decrease in kinetic energy of the photoelectrons on an XPS spectrum. Additional satellites

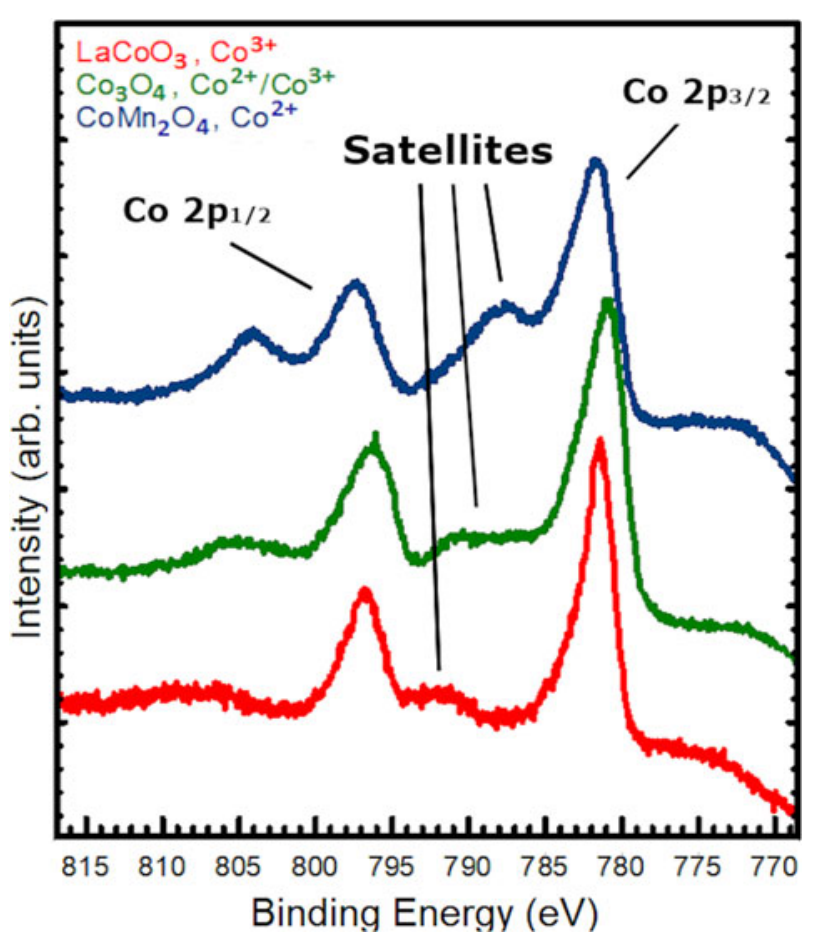

Figure 4: $\mathrm{Co} 2 p$ XPS data for $\mathrm{LaCoO}_{3}, \mathrm{Co}_{3} \mathrm{O}_{4}$, and $\mathrm{CoMn}_{2} \mathrm{O}_{4}$ with varying $\mathrm{Co}$ valences.

created by charge transfer can appear as shoulders on primary peaks or as completely distinct peaks a few $\mathrm{eV}$ away from the primary peak. An important thing to note is that both the $j=1 / 2$ and $j=3 / 2$ each have a corresponding satellite and that the ratio of intensity of $2: 1$ is still maintained for the satellites. Charge transfer satellites are observed in Ti $2 p$ and O $1 s$ spectra for materials such as $\mathrm{SrTiO}_{3}$ [77].

Shake-up and charge transfer satellite peeks should not be confused with the different valence peaks. In ionic systems such as oxides, changes of the valence state of ions are expected. When ions lose or gain valence electrons, the effective nuclear charge is changed as well and can be intuitively understood using a charged-shell model introduced by Fadley et al. [78] For positively charged cations, this change is typically on the order of a few $\mathrm{eV}$ to higher binding energies though in some cases small enough to be difficult to resolve ( $\sim 0.5 \mathrm{eV}$ or less). This can be a very powerful tool to identify the bonding environment of atoms in a material, but also makes data analysis significantly more complicated and is often incorrectly presented in the literature. As an example, we show three distinct spectra for different Co valences acquired in our lab in Fig. 4. Co is an example of an ion that does not exhibit significant differences in chemical shift between the $2+$ and $3+$ charge state. Naive fits to the data will generally not be sufficient to accurately determine the ratio of $\mathrm{Co}^{2+}$ to $\mathrm{Co}^{3+}$ in a mixed-valence material. Instead, readers are encouraged to refer to literature guides for rigorous fitting procedures [70]. The Co $\mathrm{L}_{3} \mathrm{M}_{23} \mathrm{M}_{45}$ Auger 


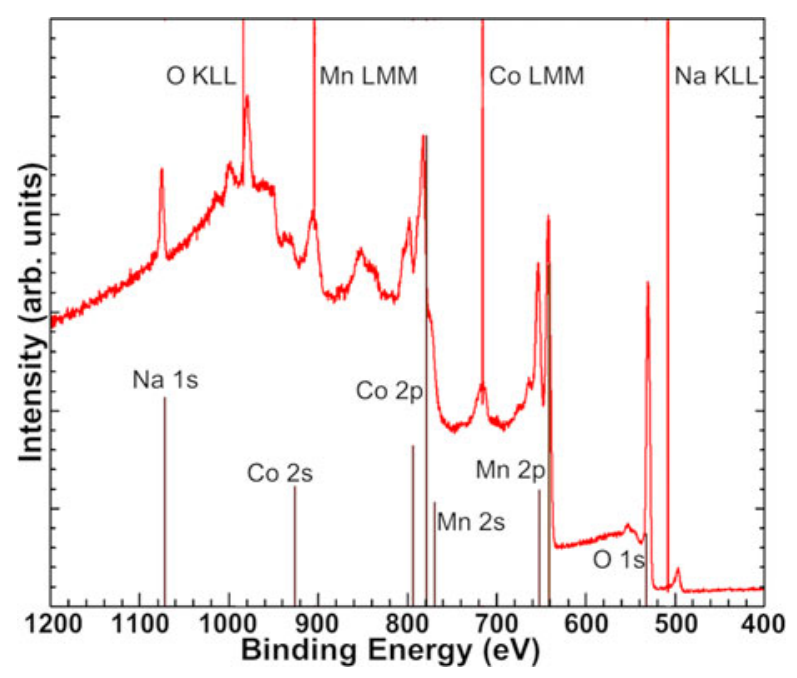

Figure 5: XPS survey of $\mathrm{CoMn}_{2} \mathrm{O}_{4}$ thin film showing $\mathrm{Na}$ contamination from oxygen plasma source.

electron also generates peak intensity at $\sim 777 \mathrm{eV}$, accounting for the shoulder at low binding energy below the Co $2 p_{3 / 2}$ peak, which can complicate quantification and peak fitting.

\section{Combining growth and XPS to measure film quality}

\section{Measuring contaminants}

Epitaxial thin film research has a very low tolerance for elemental contamination. Despite engineering controls, contaminants from unknown sources sometimes make their way into samples and can either hinder data acquisition in experiments or change the physical properties of the material entirely. Surface-sensitive XPS is an excellent way to detect any unwanted element contamination, with an elemental sensitivity of $<1$ atomic percent. The most efficient way to measure contamination is by acquiring wide-range surveys of samples from maximum binding energy to zero. In general, it is good practice to perform surveys on every sample before performing a longer-duration, high-resolution measurement over core level and valence band regions. XPS surveys allow for a quick qualitative depiction of a sample's surface stoichiometry in addition to indicating any unexpected elemental contaminants.

As an example, Fig. 5 is a survey of a $\mathrm{CoMn}_{2} \mathrm{O}_{4}$ spinel thin film sample grown in our MBE system. There are many peak features in the survey representing cobalt, manganese, and oxygen signals, but additional peaks indicate a contaminating element. Based on the position and shape of these extra features, we determined the presence of sodium on the sample surface. By inspecting surveys of samples grown at different conditions, we pinpointed the source of the sodium to the radio-frequency oxygen plasma source. After discussions with the manufacturer, the Na-contaminated quartz discharge tube was replaced with an alumina discharge tube, which has eliminated contamination. We are aware of at least one other plasma source that has also been a source of $\mathrm{Na}$ contamination and suggest that groups should be careful to test new plasma sources upon purchase. Given that the design employed by the manufacturer (Mantis Deposition) has been ubiquitous in the field for many years, we suspect that other groups without access to in situ XPS may have $\mathrm{Na}$ contamination in films without realizing. Other contaminants can be detected by XPS, such as F due to surface treatments performed on $\mathrm{SrTiO}_{3}$ with a buffered oxide etchant [79]. In this sense, the availability of in situ XPS can accelerate the calibration process for ideal growth conditions and pristine materials, making it valuable as a day-to-day diagnostic tool even when the data will not be used for publishable research.

\section{Film stoichiometry and surface termination}

In practice, the depth sensitivity of XPS can be both a blessing and a curse. The angle-resolved XPS technique can be used to measure the surface termination of a sample, cation intermixing across an interface, and determine a depth profile of specific chemical features, providing rapid new depth-resolved insights into synthesized materials without destroying the sample through sputter etching or focused ion beam liftout for electron microscopy measurements. Conversely, the surface sensitivity of XPS means that a change as small as flipping the surface termination from $\mathrm{TiO}_{2}$ to $\mathrm{SrO}$ on a $\mathrm{SrTiO}_{3}$ single crystal can reduce the measured $\mathrm{Ti}: \mathrm{Sr}$ peak area ratios by $\sim 10 \%$. This makes stoichiometry quantification for epitaxial thin films by XPS very difficult, with conventional sensitivity factors such as those used in XPS data analysis software providing very little value. Further consideration must be given to the effects of X-ray photoelectron diffraction, which can change relative peak intensities due to forward scattering of photoemitted electrons from the single crystal surface. It is generally best to benchmark the peak area ratios with a bulk-sensitive technique such as Rutherford back scattering on a film grown to produce a known surface termination. Alternatively, one can measure a single crystal reference with a known surface termination such as a treated $\mathrm{SrTiO}_{3}$ substrate [80]. After these initial tests, is it then possible to accurately compare peak area ratios for subsequent films to the measured area ratios from the calibrated reference sample while taking care to only compare samples with the same surface terminations.

However, despite the challenges of absolute stoichiometry quantification via XPS, we have found a convenient trick that employs in situ capabilities to accelerate the calibration process for oxide MBE growth. Given the layered nature of the perovskite crystal structure and the propensity for excess cations to reside on the film surface [81, 82], using angle-resolved XPS to determine the surface termination is generally an effective 
means to determine whether a perovskite oxide film has excess A-site or B-site cations even without an RBS or single crystal reference standard. The segregation is shown schematically in Figs. 6(a) and 6(b), with excess $\mathrm{TiO}_{2}$ and $\mathrm{SrO}$ residing on the film surface. Note that this figure is meant to schematically portray clustering of excess atoms on the film surface and not a particular surface reconstruction that might occur in such a case. The angle-resolved XPS measurement process involves comparing peak area ratios for the A-site ion to the B-site ion in a bulksensitive configuration near-normal emission $\left(\theta<45^{\circ}\right)$ and in a surface-sensitive configuration $\left(\theta>70^{\circ}\right)$. Such measurements can generally be completed in under an hour, making it possible to estimate whether a sample is A-site or B-site rich and recalibrate prior to additional depositions. For example, in the case of $\mathrm{SrTiO}_{3}$ films, we have seen that the ratio of the Ti $2 p$ to the Sr $3 d$ peak areas increases for larger values of $\theta$ if the sample is Ti-rich [83]. A comparison of one such sample is shown in Fig. 6(c). In this case, the bulk and surface-sensitive Sr $3 d$ and Ti $2 p$ peaks have been normalized so that the $\mathrm{Ti} 2 p$ peaks show the same total peak area, which highlights the smaller peak area for the surface-sensitive Sr $3 d$ peak relative to its bulk counterpart. An additional source of information is the presence of surface core-level shifts that can be measured due to incomplete coordination or $\mathrm{OH}$ adsorption of surface A-site ions, as has been shown for Ba-terminated $\mathrm{BaTiO}_{3}$, which shows a distinct peak at higher binding energy for the $\mathrm{Ba} 3 d$ peak [84]. Using these approaches while understanding the kinetics of the growth process can make in situ XPS particularly valuable for quick tuning of growth parameters sample-to-sample.

Beyond the convenience of angle-resolved XPS in the synthesis process, it has helped to explain surface-dependent phenomena in a variety of systems. We have employed angle-resolved XPS to determine for the first time that an $\mathrm{SrO}$ termination is more stable for stoichiometric $\mathrm{SrTiO}_{3}$ films grown by hybrid MBE [83]. Furthermore, in the case of a $\mathrm{LaAlO}_{3} / \mathrm{SrTiO}_{3}$ heterostructure grown on SrO-terminated $\mathrm{SrTiO}_{3}$, an unexpected $\mathrm{AlO}_{2}$ termination was observed by angle-resolved XPS, which helped to explain the absence of conductivity at the interface [85]. Angle-resolved measurements of $\mathrm{LaFeO}_{3}$ film surfaces have also helped to explain the role of surface termination on chemical reactivity for catalytic water splitting [86]. Despite the simplicity of angle-resolved XPS and the relatively mundane knowledge gleaned from knowing the surface termination of a thin film, there is a great deal of new physical understanding that can arise from such day-to-day measurements through in situ XPS.

\section{Oxide surface chemistry}

While understanding of the surface termination regarding perovskite $\mathrm{AO}$ or $\mathrm{BO}_{2}$ layers is valuable for film synthesis, it

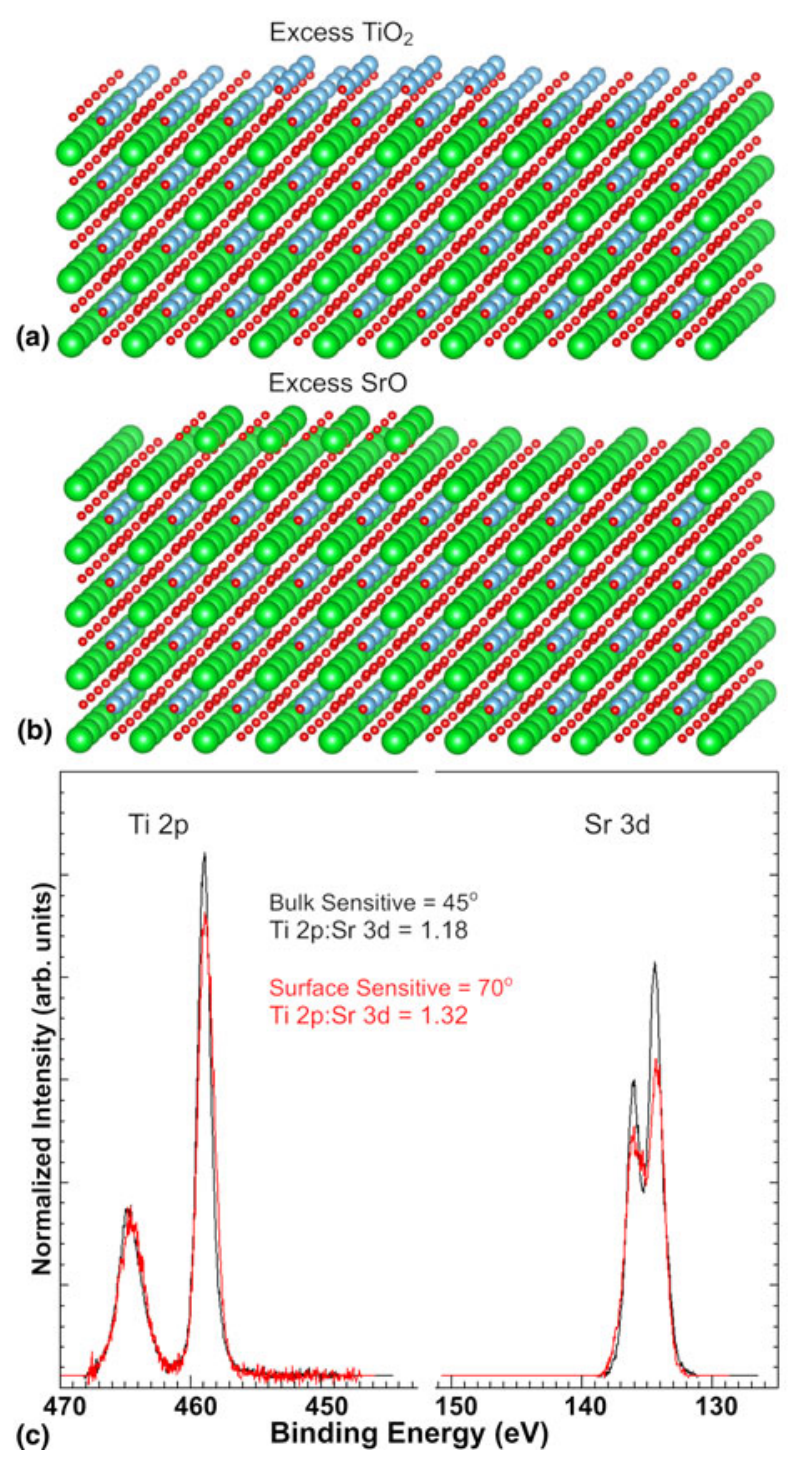

Figure 6: (a) Model of excess $\mathrm{TiO}_{2}$ on the film surface of $\mathrm{TiO}_{2}$-terminated $\mathrm{SrTiO}_{3}$, (b) model of excess $\mathrm{SrO}$ on the film surface of SrO-terminated $\mathrm{SrTiO}_{3}$, and (c) angle-resolved XPS measurement of off-stoichiometric $\mathrm{SrTiO}_{3}$ film with excess Ti cations at the film surface.

only begins to address the subtleties of surface chemistry that occur in complex oxides. The measurement of the $\mathrm{O} 1 \mathrm{~s}$ peak can also provide valuable information to understand how the surface is passivated, a process that occurs in different ways depending on the surface polarity. It is known that water will adsorb differently depending on the $\mathrm{AO}$ or $\mathrm{BO}_{2}$ termination of a (001)-oriented surface $[83,86]$. However, even in UHV after in situ growth, the adsorption of water on the surface can impact the electronic properties of the material. Studies that integrate XPS with atomically resolved STM are incredibly valuable in this regard to demystify some of the surface phenomena that drive emergent behavior.

As discussed above, the $\mathrm{KTaO}_{3}$ surface 2DEG is of significant interest for the Rashba splitting that is observed [33]. 
Through STM and XPS studies, Setvin et al. demonstrated a $2 \times 1$ surface reconstruction of the surface with uniform $\mathrm{K}$ $(\mathrm{OH})_{2}$ coverage after water exposure [38]. An $\mathrm{OH}$ peak on the $\mathrm{O} 1 s$ core level at higher binding energy was reported for this case to confirm their results. Similar experiments have also been performed on layered ruthenates $\left(\mathrm{Ca}_{3} \mathrm{Ru}_{2} \mathrm{O}_{7}\right.$ [87] and $\mathrm{Sr}_{2} \mathrm{RuO}_{4}$ [88]) and show that water can adsorb onto the surfaces of oxides in a variety of different configurations with different binding energies relative to the metal oxide $\mathrm{O} 1 \mathrm{~s}$ peak that is typically at $\sim 530.0 \mathrm{eV}$. It is generally observed that films with $\mathrm{AO}$ termination will more readily adsorb water $[38,83,86,88]$, though the exact surface structure is very difficult to decipher. The effect of the adsorbed watereven after in situ growth-should not be discounted when analyzing the observed properties. For example, it is common in the literature to associate a higher binding energy peak $2.3 \mathrm{eV}$ above the metal oxide binding energy with oxygen vacancies, but the origin of this peak is the subject of some controversy and it can easily be due to other surface chemistry effects that will complicate the analysis [86].

\section{Probing interfacial phenomena in oxide heterostructures with XPS}

\section{Interfacial chemistry and intermixing}

The surface sensitivity of XPS through angle-resolved measurements can be extremely useful for the purposes of determining depth-dependent phenomena in oxide heterostructures. We have already showed the effects of surface variations and how they can be used to understand oxide film growth. However, the same techniques can be taken a step further to understand such behavior as interfacial intermixing in heterostructures and growth-induced defects such as oxygen vacancies. By varying the photoelectron emission angle to the detector and modeling the angular dependence of the relative peak areas based on the IMFP for the sample, one can construct a quantitative model of the cation or defect profile as a function of depth within a material.

For a buried interface, angle-resolved measurements can be used to model the depth of defects or dopants. The approach has been particularly valuable in studies of polar/non-polar interfaces, where differences between the idealized physical model of an interface and the actual synthesized heterostructure have led to confusion as to the physical origin of emergent behavior. In the case of $\mathrm{LaAlO}_{3} / \mathrm{SrTiO}_{3}$ interfaces, angle-resolved measurements have shown that cations from the underlying $\mathrm{SrTiO}_{3}$ substrate will readily out-diffuse into the $\mathrm{LaAlO}_{3}$ film, with the degree of diffusion dependent on the cation stoichiometry of the $\mathrm{LaAlO}_{3}$ film $[19,89]$. Through rigorous Monte Carlo modeling of the angle-resolved data, one can even estimate the cation concentration profile across the interface in a heterostructure, as Salvinelli and colleagues have demonstrated [90]. These measurements set the stage for further studies that confirmed that the 2DEG at these interfaces is dependent on the film stoichiometry [20, 21] and thus driven in part by the formation of interfacial defects such as oxygen vacancies. Angle-resolved measurements can also be used to model the depth of distinct chemical and electronic states, such as the $\mathrm{Ti}^{3+}$ states at the $\mathrm{LaAlO}_{3} / \mathrm{SrTiO}_{3}$ interface. By probing the Ti $2 p$ core level of samples using ex situ hard X-ray photoemission, Sing et al. developed a model of the thickness of the 2DEG in UCs to show that it was confined to a single UC at the surface of the $\mathrm{SrTiO}_{3}$ substrate [91]. The results from this work are shown in Fig. 7. Other angle-resolved studies have shown that oxygen vacancies in $\mathrm{SrTiO}_{3}$-based heterostructures are localized at the interface $[92,93]$.

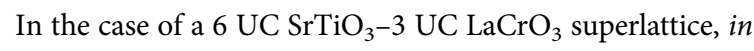
situ angle-resolved XPS measurements were used to determine the degree of $\mathrm{Cr}$ out-diffusion into the topmost $\mathrm{SrTiO}_{3}$ layer [94]. The resulting data were compared with a model with differing degrees of $\mathrm{Cr}$ intermixing to estimate the $\mathrm{Ti}-\mathrm{Cr}$ concentration profile across the B-site of the superlattice. Interfacial models were constructed to represent differing percentages of B-site cation intermixing and contributions to the overall $\mathrm{Cr}$ $2 p$ and Ti $2 p$ peak intensities were estimated by accounting for the IMFP in the material for each layer. The resulting estimates showed that there is significant intermixing of $\mathrm{Ti}$ across the interface, with approximately $25 \%$ of the middle B-site ions in the $\mathrm{LaCrO}_{3}$ layer occupied by Ti ions. Critically, the model agreed very well with extracted concentration profiles from STEM-EELS measurements, as shown in Fig. 8 [94].

\section{Band alignment and potential gradients}

Semiconductor heterostructures are key to modern-day electronics, and numerous oxide heterojunctions have been pursued for their potential applications. The performance of these heterostructures can be engineered by manipulating valence and conduction band offset at the interface. It is crucial to understand this band offset to accurately predict and manipulate the behavior of thin film heterostructures.

There are different schemes to determine the band offset experimentally such as ultra-violet spectroscopy, internal photoemission spectroscopy, and XPS. Band offset determination via XPS has been used extensively. Unlike other methods, XPS is sensitive to details at the interface such as changes in valence and chemical intermixing and thus is often preferred over other methods. The XPS approach to valence band alignment determination was first introduced by Kraut et al. [95]. It is based on the premise that the energy difference between a 


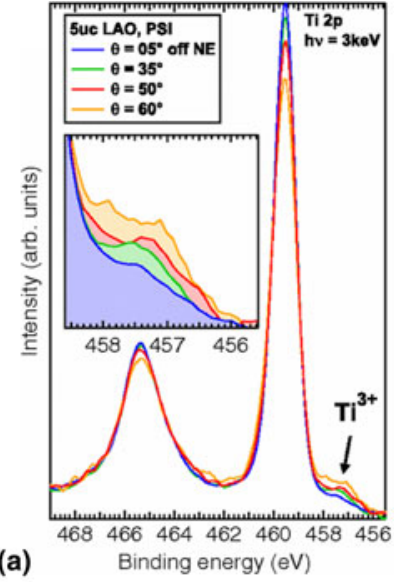

(a)

(b)
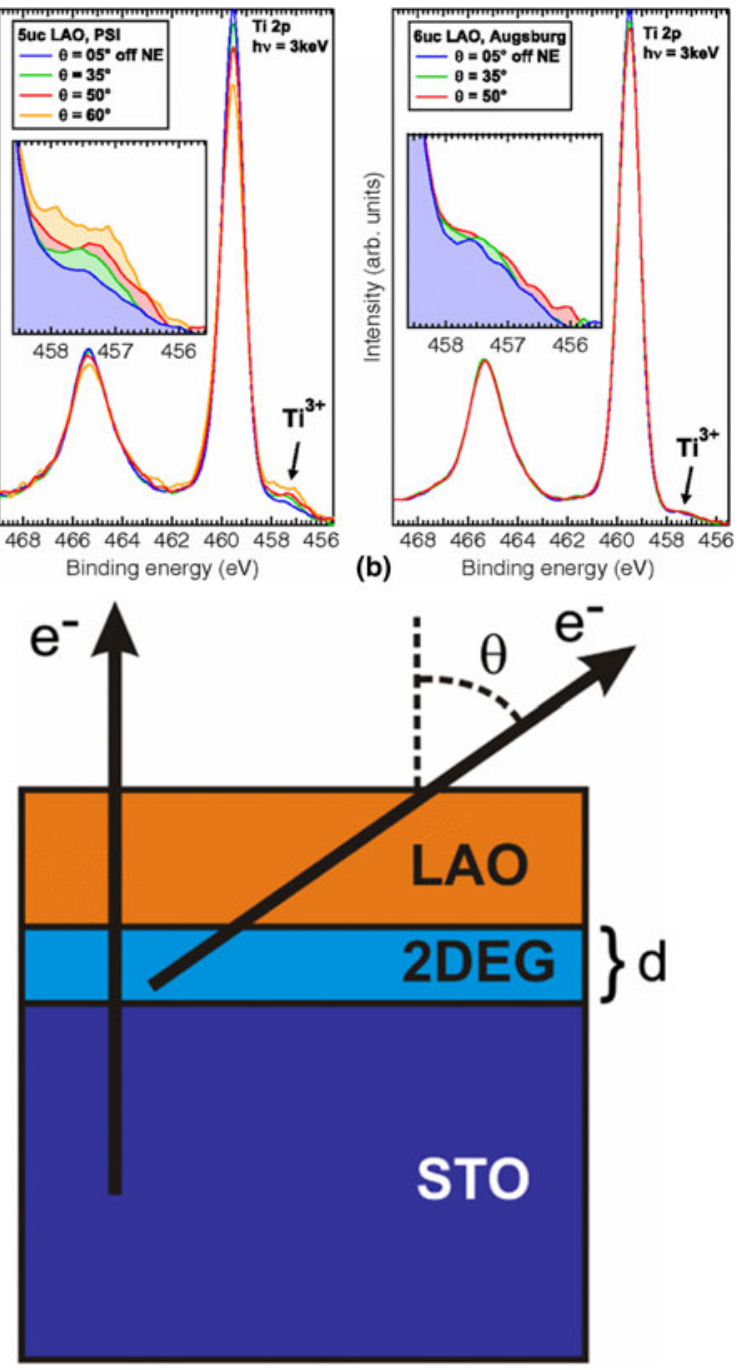

Figure 7: $\mathrm{Ti} 2 p$ core-level data showing $\mathrm{Ti}^{3+}$ intensity at the $\mathrm{LaAlO}_{3} / \mathrm{SrTiO}_{3}$ interface for two different samples (a and b). Variation with angle in (a) shows that $\mathrm{Ti}^{3+}$ is non-uniformly distributed in comparison to the uniform distribution in (b). (c) Schematic of angle-resolved XPS measurement of 2DEG location. Reprinted figure with permission from Sing et al. [91]. Copyright 2009 by the American Physical Society.

core level and valence band maximum (VBM) is an intrinsic property and remains constant independent of any formation of a heterostructure. Any change in the binding energy of the VBM will change the core level equally, making the energy of the core-level peak a good proxy for the VBM. This becomes important when measuring a thin film heterostructure, as the valence band region will now be the convolution of two or more distinct materials, while appropriately chosen core levels can still be measured easily and fit repeatably. The precision of this method lies in the measurement of core level and valence band maxima binding energies from reference samples for each material in a heterojunction. This can be accomplished using a single crystal reference or carefully grown film that is sufficiently thick such that the underlying substrate does not

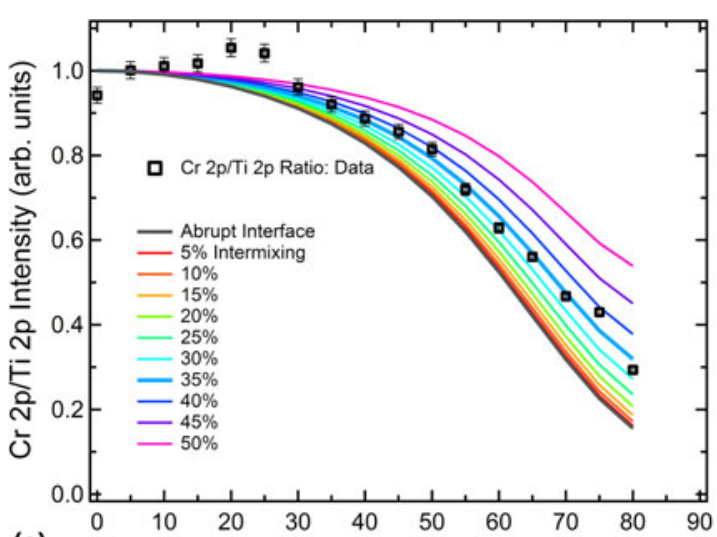

(a) Takeoff Angle (deg, relative to normal emission)

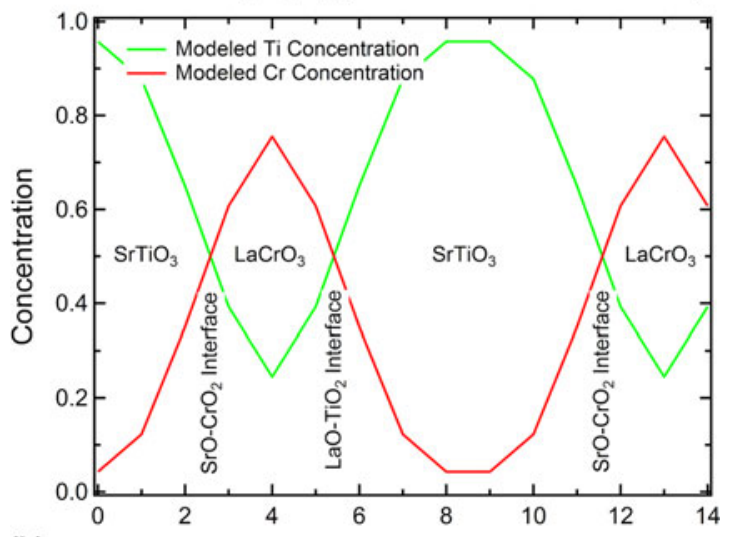

(b)

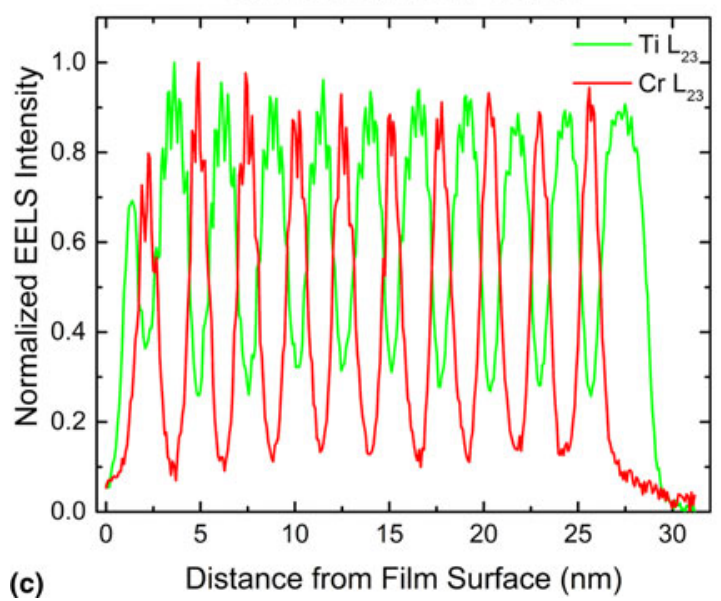

Figure 8: (a) Angle-resolved XPS Cr $2 p: T i 2 p$ peak ratio with models assuming various degrees of intermixing $\left(0^{\circ}\right.$ is normal to the film surface), (b) concentration profile for the 35\%-intermixing model, and (c) STEM-EELS integrated signal profile throughout superlattice determined using MLLS fitting of the $\mathrm{Cr} \mathrm{L}_{23}$ edge and the background-subtracted peak area of the $\mathrm{Ti}_{23}$ edge. The signal has been normalized to the substrate. Reprinted with permission from Comes et al. [94]. Copyright 2017 American Chemical Society.

contribute any signal to the data. With the two reference samples and one more heterojunctions under examination, at least three samples are needed to apply this approach.

Using their data, Kraut et al. [95] determined the VBM by fitting valence band spectra with the theoretical valence band 
density of states. However, fits involving a linear extrapolation of the leading edge of the valence band spectra to the zero-level backgrounds have been found to be as accurate and require much less effort. Various refinements to this approach over the years for oxide heterostructures have been proposed [96, 97]. A diagram illustrating Kraut's method is shown in Fig. 9. The valence band and conduction band offset are calculated as

$$
\begin{aligned}
& \Delta E_{\mathrm{v}}=\left(E_{\mathrm{CL}}^{\mathrm{A}}-E_{\mathrm{V}}^{\mathrm{A}}\right)-\left(E_{\mathrm{CL}}^{\mathrm{B}}-E_{\mathrm{V}}^{\mathrm{B}}\right)-\left(E_{\mathrm{CL}}^{\mathrm{A}}-E_{\mathrm{CL}}^{\mathrm{B}}\right), \\
& \Delta E_{\mathrm{C}}=E_{\mathrm{g}}^{\mathrm{A}}-E_{\mathrm{g}}^{\mathrm{B}}+\Delta E_{\mathrm{V}} .
\end{aligned}
$$

The core-level peaks and valence band reference spectra should be acquired with high energy resolution, as the uncertainty of the measurement of the core-level peak position will propagate into the band offset calculation. The choice of peaks that have a narrow intrinsic width and are easier to fit repeatably is thus very important.

The $\mathrm{LaAlO}_{3} / \mathrm{SrTiO}_{3}$ interface has been widely studied for its unusual transport properties. The properties can be attributed to an effective 2DEG at the interface. The origin of this 2DEG has been the source of great controversy, with some attributing it to the polar discontinuity $[1,91]$ and others to defect-driven phenomena $[19,20,21,23]$. Owing to its high precision in measuring core levels and VBMs, and subsequently the valence band and conduction band offsets, XPS has been a powerful tool in studying this heterostructure interface. Additionally, XPS data can be modeled to estimate the potential gradient across the film.

Multiple groups have used XPS to study the $\mathrm{LaAlO}_{3} / \mathrm{SrTiO}_{3}$ interface grown by both MBE [22] and PLD [19, 98, 99] with different film thicknesses and terminations for possible explanations for the origin of the interface's 2DEG. In one such work, Segal et al. confirmed the metal-insulator interface transition with $4 \mathrm{UC}$-thick $\mathrm{LaAlO}_{3}$ on a $\mathrm{TiO}_{2}$-terminated surface [22]. Many studies suggest that the 2DEG at the interface stems from an electronic reconstruction due to polar discontinuity (LAO has alternating positive and negative atomic layers), known as the "polar catastrophe" [91]. From the experimental band offset and band gap, Segal et al. calculated that a potential gradient of $1.15 \pm 0.06$ at a 4 UC thickness is required for the polar catastrophe to occur, whereas the measured potential gradient at 4 UCs was much less than the required value. This means that other phenomena such as oxygen defects, lattice distortion, and cation mixing at the interface must be investigated to explain this unique behavior at the interface. In this way, XPS has helped to further elucidate the origin of 2DEG at the LAO-STO interface, which was later shown to be strongly dependent on $\mathrm{LaAlO}_{3}$ cation off-stoichiometry [20, 21].

Other studies of interfaces where both sides of the heterojunction are comprised of band insulators with the VBM comprised of $\mathrm{O} 2 p$-derived electronic states, have generally shown

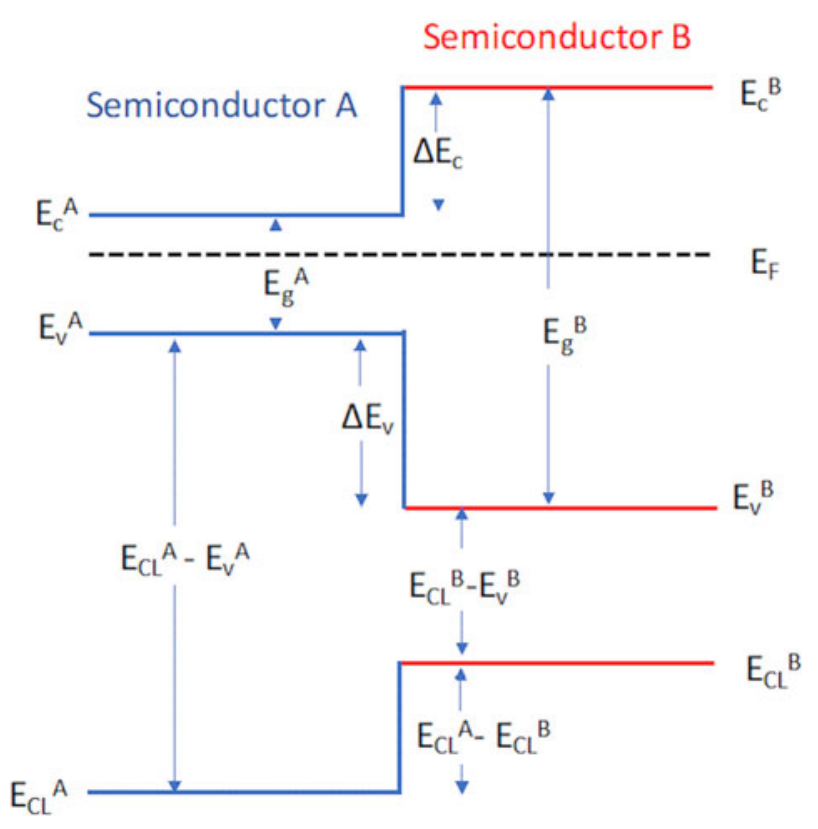

Figure 9: Schematic rendering of method to measure band alignment in XPS via core-level binding energies. $E_{\mathrm{CL}}$ refers to core-level binding energy, $E_{\mathrm{V}}$ refers to the $\mathrm{VBM}$, and $E_{\mathrm{C}}$ refers to the conduction band minimum for materials $A$ and $B$. By measuring these values for thick films or single crystal references, one can then determine the value of $\Delta E_{\mathrm{V}}$ for a heterostructure by measuring the difference $E_{\mathrm{CL}}^{\mathrm{A}}-E_{\mathrm{CL}}^{\mathrm{B}}$.

small valence band offsets of $\sim 0.5 \mathrm{eV}$ or less [100]. This includes the $\mathrm{SrTiO}_{3} /(\mathrm{La}, \mathrm{Sr})(\mathrm{Al}, \mathrm{Ta}) \mathrm{O}_{3}$ interface [101], the $\mathrm{SrZrO}_{3} / \mathrm{SrTiO}_{3}$ interface [102], and the $\mathrm{BaSnO}_{3}$ interface with both $\mathrm{SrTiO}_{3}$ and $\mathrm{LaAlO}_{3}$ [103]. The small valence band offset can generally be attributed to the condition that the $\mathrm{O} 2 p$ energy level is continuous across the heterojunction [104], which will be discussed in more detail shortly.

Unlike the isoelectronic interfaces where $\mathrm{O} 2 p$ states form the top of the valence band on both sides of the heterojunction, interfaces between Mott-Hubbard and band insulators offer additional degrees of freedom. For example, the $\mathrm{LaFeO}_{3} /$ $n$ - $\mathrm{SrTiO}_{3}$ system also creates a polar non-polar interface and is studied for its photocatalytic applications [105, 106, 107, 108]. Nakamura et al. [105] studied the interface-induced polarization of $\mathrm{LaFeO}_{3} / \mathrm{Nb}$-doped $\mathrm{SrTiO}_{3}$ heterostructures and observed novel shift current that suggested the polarization direction switches for different substrate terminations ( $\mathrm{SrO}$ and $\mathrm{TiO}_{2}$ ). This would mean that potential gradients took on opposite signs for different terminations and suggested that changes in polarization are responsible for observed photoconductivity. Subsequent studies of $\mathrm{LaFeO}_{3} / \mathrm{Nb}$-doped $\mathrm{SrTiO}_{3}$ junctions with varying $\mathrm{LaFeO}_{3}$ film thicknesses (3,6 and $9 \mathrm{UCs}$ ) and different substrate terminations $\left(\mathrm{SrO}\right.$ and $\mathrm{TiO}_{2}$ ) grown using $\mathrm{MBE}$ and characterized by in situ XPS helped to address these questions [107]. These studies found an increase in separation between core levels ( $\mathrm{La} 4 d$ and $\mathrm{Sr} 3 d$ ) with increases in film thickness, clearly indicating that band offset and built-in potential change 

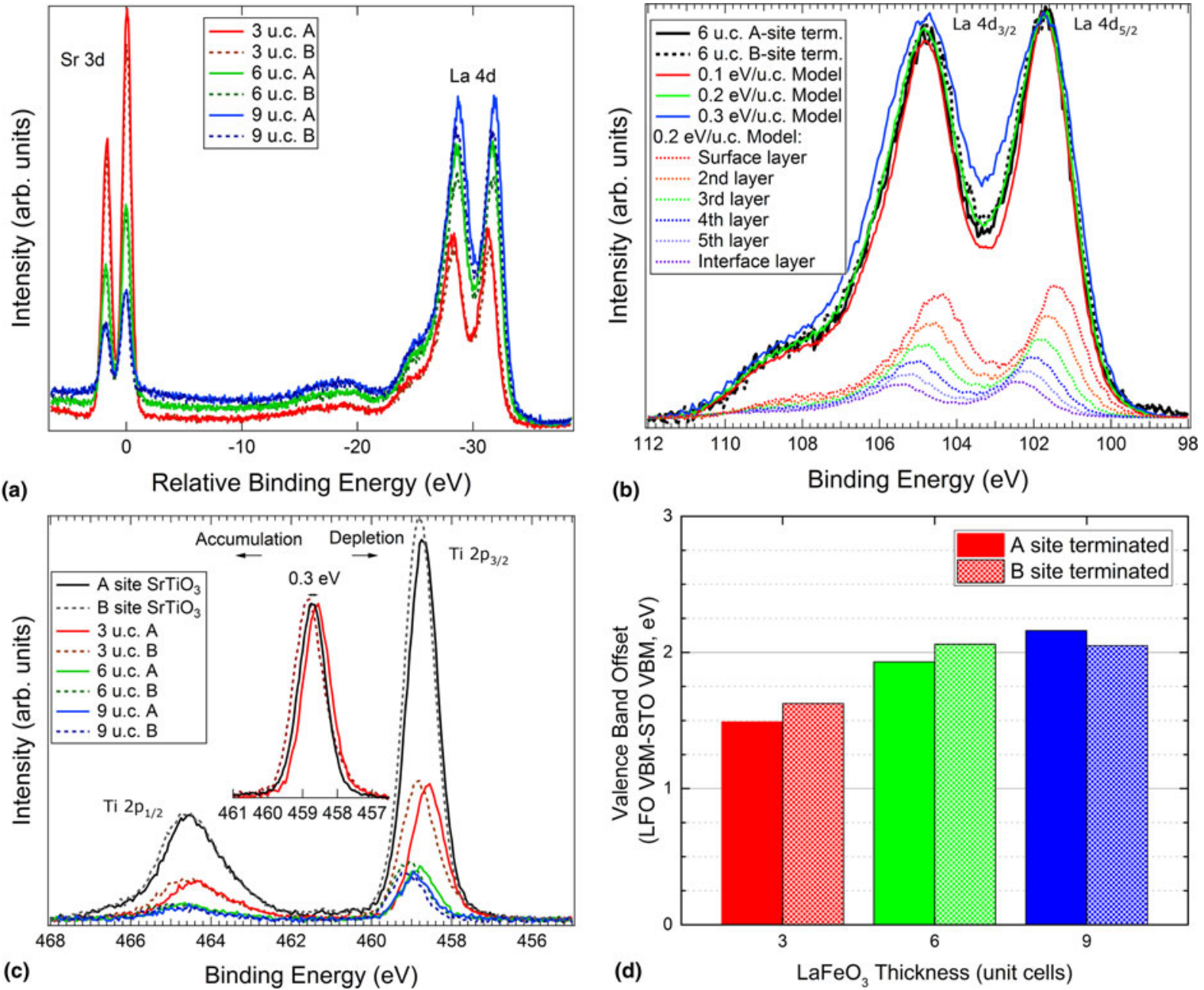

Figure 10: (a) Sr $3 d$ and La $4 d$ core-level spectra for the family of heterostructures, shifted to align the $\mathrm{Sr} 3 d$ peaks; (b) model of La $4 d$ peak broadening in the 6 u.c. films; (c) Ti $2 p$ core-level spectra for each film and substrate, with the inset showing the peak shifts; (d) valence band offsets determined from the core-level spectra for each heterojunction. Reprinted figure with permission from Comes and Chambers (2016) [107]. Copyright 2016 by the American Physical Society.

with thickness. In addition, by modeling the broadening of the La $4 d$ peaks with different film thicknesses and terminations, it was possible to estimate the potential gradient across the film. Contrary to the reported interface-induced polarization [105, 106], the results indicated that the interface termination had only a small impact on the band alignment and the built-in potential gradient [107]. The models are shown in Fig. 10. The chemical instability of the $\mathrm{SrO} / \mathrm{FeO}_{2}$ interface has been proposed as a likely explanation for these results [109]. These results were subsequently matched by first-principles band alignment models of the interface [108].

\section{Interfacial charge transfer}

As in modulation-doped semiconductor heterostructures, interfacial charge transfer is an important tool in complex oxides to induce novel magnetic and electronic functionalities that do not occur in equilibrium. One of the crucial parameters to determine the charge transfer probability is the charge transfer gap. In most of the cases, the charge transfer gap is determined by band splitting of B-site cations or the band gap between B-site cations and fully occupied $\mathrm{O} 2 p$ bands, while A-site cations have a less significant role. Predicting charge transfer is not straight forward in oxide heterostructures since the classic band alignment principle is not strictly followed as in the case of semiconductors. A modified rule is based on the continuity of states of the $\mathrm{O} 2 p$ band across the interface and allows for a qualitative prediction of band alignments and charge transfer in complex oxide heterostructures [104]. In cubic oxides, the local energy of O $2 p$ states relative to the Fermi level, $\varepsilon_{\mathrm{p}}-E_{\mathrm{F}}$, has been proposed to be the deterministic factor to tune the direction of charge transfer from one complex oxide to another. Generally, electron transfer favors electron flow from lower (more negative) $\varepsilon_{\mathrm{p}}-E_{\mathrm{F}}$ to higher. The classic semiconductor rule is shown in Fig. 11(a), while the modified rule for oxides is shown in Figs. 11(b)-11 
(a)

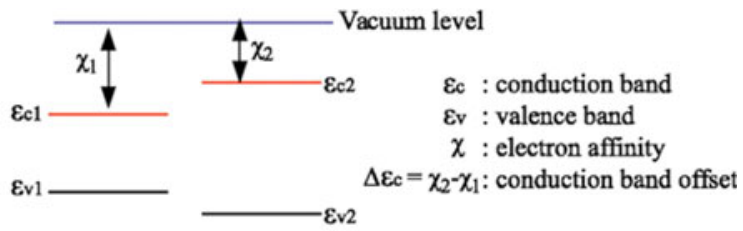

(b)

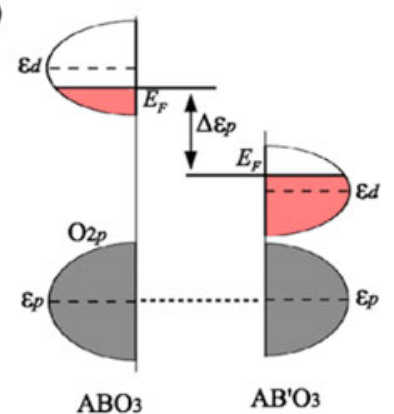

(d)

$\mathrm{ABO}_{3}$

$\mathrm{AB}^{\prime} \mathrm{O}_{3}$

(c)

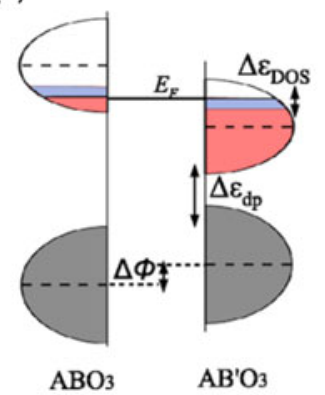

(e)

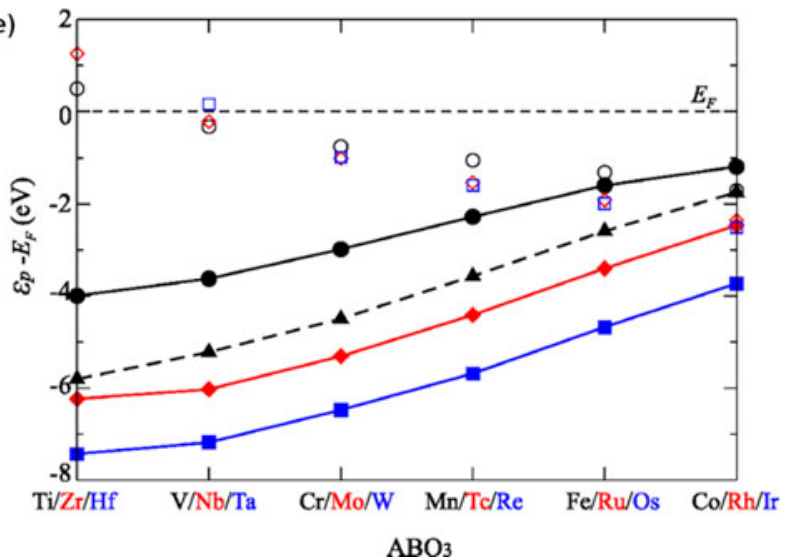

Figure 11: (a) Conventional model for semiconductor band alignment based on aligning the vacuum level for constituent materials; (b) preliminary band alignment based on the alignment of $02 p$ states prior to charge transfer; (c) reconstructed band alignment after charge transfer equilibration of the Fermi energy level; (d) schematic model of $\mathrm{ABO}_{3} / \mathrm{AB}^{\prime} \mathrm{O}_{3}$ interface emphasizing continuity of $\mathrm{O} 2 p$ electronic states; (e) summary of bulk $\varepsilon_{p}$ (filled symbols) and $\varepsilon_{\mathrm{d}}$ (empty symbols) with respect to the Fermi level $\left(E_{\mathrm{F}}=0\right)$ for different $\mathrm{SrBO}_{3}$ (solid line) materials, with $3 d$ (black), $4 d$ (red), and $5 d$ (blue) elements. The simple criterion for the direction of the charge transfer at the $\mathrm{ABO}_{3} / \mathrm{AB}^{\prime} \mathrm{O}_{3}$ interface is that the component with lower (more negative) $\varepsilon_{\mathrm{p}}-E_{\mathrm{F}}$ will donate electrons across the interface to the opposite material. Also plotted is $\mathrm{LaBO}_{3}$ (dashed line) for $\mathrm{B}=3 d$, for estimates for $\mathrm{ABO}_{3} / \mathrm{A}^{\prime} \mathrm{BO}_{3}$ interfaces. Adapted under Creative Commons Attribution 3.0 License from Zhong and Hansmann [104].

(e). This rule led to the development of alternative computational methods to predict charge transfer that have been shown to be effective in recent years.

Density functional theory (DFT) is one of the most widely used theoretical modeling techniques to predict the charge transfer mechanism in the metal oxides interface. Different metal oxides interfaces follow different charge transfer mechanisms. Particularly in Mott-Hubbard insulators, the O $2 p$ band alignment and the competition between crystal field and correlation energy of $d$ electrons are crucial to determine the electronic rearrangement [104]. Generally, the apical oxygen atom at the interface [see Fig. 11(d)] is shared by the materials at the heterostructure interface. This results in the alignment of O $2 p$ bands at the interface. However, considering the $\mathrm{O} 2 p$ band alignment alone disregards the creation of the internal electric field that balances the electrochemical potential between two B-sites and prevents further charge transfer. Hence, interfacial charge transfer between two materials transfer cannot completely be understood relying only on $\mathrm{O} 2 p$ band alignment. An extra factor that comes into play is the rearrangement of the $d$ - bands on B-site cations. Specifically, the density of states of B-site cations of each material reflects a clear picture of possible electronic rearrangement.

As an example, the $\mathrm{Ti}$ atom would be expected to donate electrons to the $\mathrm{Fe}$ atom at the $\mathrm{LaTiO}_{3} / \mathrm{LaFeO}_{3}$ interface, implying a formal charge of $4+$ for the interfacial $\mathrm{Ti}$ ion and the shifting of Fe from a 3+ state to a 2+ state near the interface [104]. A theoretical prediction of charge transfer in the metal oxides interface is supported by XPS analysis both qualitatively and quantitatively to resolve the valence band structure. XPS is well known for its high sensitivity to the variations in valence states of transition metal ions. Kleibuker et al. [110] showed an extra peak at $\sim 2 \mathrm{eV}$ lower binding energy is observed for the $\mathrm{LaFeO}_{3} / \mathrm{LaTiO}_{3}$ heterointerface in reference to the bulk $\mathrm{LaFeO}_{3}$ for Fe $2 p$ XPS spectra, as shown in Fig. 12. This extra peak in Fig. 12(c) at slightly lower binding energy, denoted by an open circle, represents the presence of the $\mathrm{Fe}^{2+}$ state along with the typical $\mathrm{Fe}^{3+}$ state at higher binding energy, denoted by a solid circle. Interestingly, the extra peak gets more pronounced with the decrease in thickness of the $\mathrm{LaFeO}_{3}$ layer which indicates that the charge transfer occurs near or at the interface. To understand the rearrangement mechanism for transfer of charge, a valence band spectrum in XPS is analyzed experimentally. The evolution of an extra peak around $1 \mathrm{eV}$ in valence band spectra represents the completely filled $t_{2 \mathrm{~g}}$ band of $\mathrm{Fe}^{2+}$ in a low-spin configuration. Separate measurements of the Ti $2 p$ spectrum confirmed $4+$ formal charge of $\mathrm{Ti}$, indicative of charge transfer from $\mathrm{Ti}$ to $\mathrm{Fe}$ of $1 \mathrm{e}^{-} / \mathrm{UC}$ at the interface.

For other instances, the preparation of complex superlattices that are different from regular heterojunctions helps to enlighten the process of charge transfer. One such example is charge transfer in $\mathrm{LaTiO}_{3} / \mathrm{LaNiO}_{3} / \mathrm{LaAlO}_{3}$ heterostructures [5]. In the bulk structure, B-site cations of each material have $3+$ oxidation states. However, because the $d^{0} \mathrm{Ti}^{4+}$ is the more stable oxidation state, one electron can move from the $\mathrm{Ti} t_{2 \mathrm{~g}}$ band to $\mathrm{Ni} e_{\mathrm{g}}$ band resulting in $\mathrm{Ni}$ transitioning to a $2+$ state 
Figure 12: (a) Sketch of the $\mathrm{LaTiO}_{3} / \mathrm{LaFeO}_{3}$ sample geometry. (b) A typical $1 \times 1 \mu \mathrm{m}$ AFM height image of a $\mathrm{LaTiO}_{3} / \mathrm{LaFeO}_{3}$ heterostructure. (c) Fe $2 p$ XPS spectra of $\mathrm{LaTiO}_{3} / \mathrm{LaFeO}_{3}$ heterostructures for various thicknesses of $\mathrm{LaFeO}_{3}$, as well as of a 30 u.c. $\mathrm{LaFeO}_{3}$ film and a (2/2) $\mathrm{LaAlO}_{3} / \mathrm{LaFeO}_{3}$ heterostructure. The solid and open circles mark the $\mathrm{Fe}^{3+}$ and $\mathrm{Fe}^{2+}$ peaks, respectively. (d) Valence band XPS spectra of $\mathrm{LaTiO}_{3} / \mathrm{LaFeO}_{3}$ heterostructures for various thicknesses of $\mathrm{LaFeO}_{3}$. All spectra were taken near normal emission $\left(\theta=3^{\circ}\right)$. Reprinted figure with permission from J.E. Kleibeuker et al. [110]. Copyright 2014 by the American Physical Society.

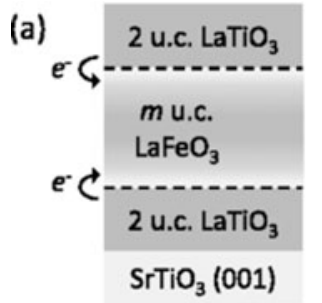

(b)
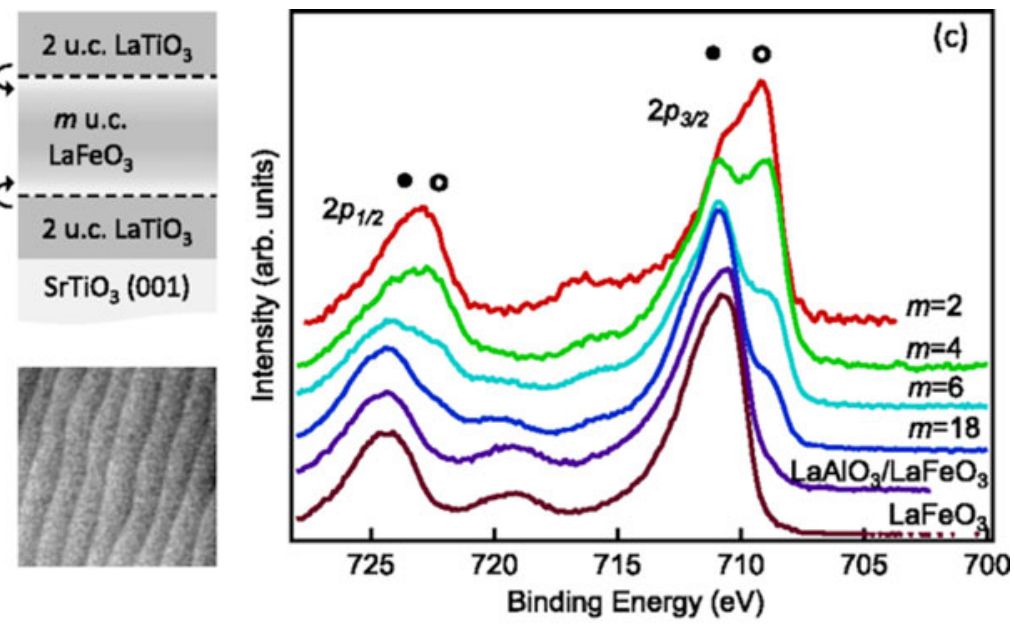

(d)

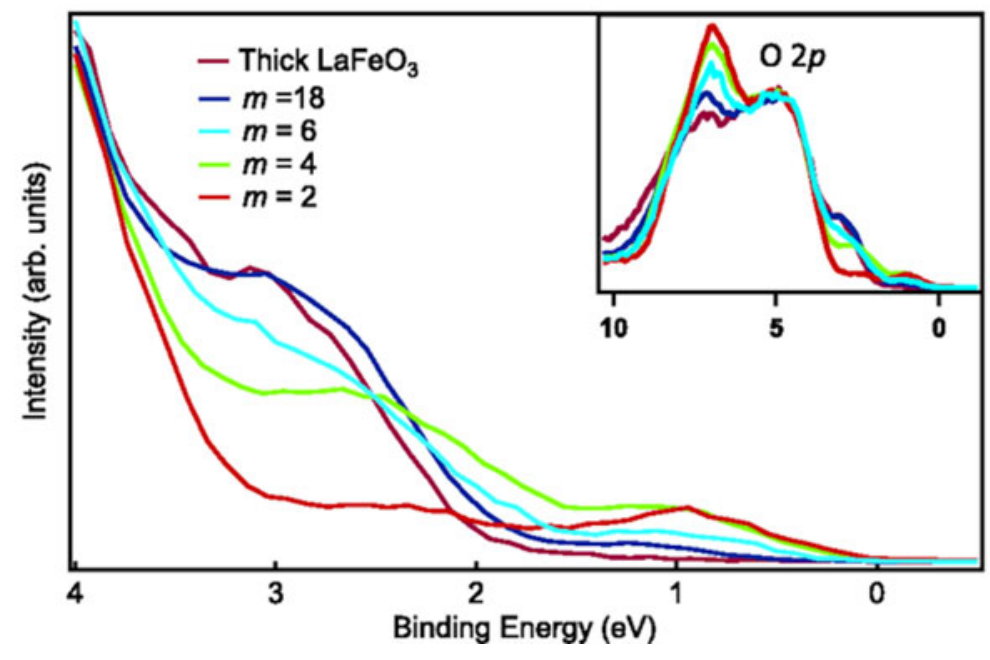

from a $3+$ state that is also more energetically favorable [5]. Interestingly, XAS confirmed this charge transfer and showed a preferential orbital occupation of the $\mathrm{Ni} 3 d_{\mathrm{z} 2} e_{\mathrm{g}}$ orbital, producing the orbital occupation that makes $\mathrm{LaNiO}_{3}$ resemble the superconducting cuprates. This type of approach to interface-engineered symmetry breaking is an alternative approach to the reduced ( $\mathrm{Nd}, \mathrm{Sr}) \mathrm{NiO}_{2}$ films that have recently demonstrated superconductivity [56]. Similar measurements for a $\mathrm{LaNiO}_{3} / \mathrm{LaMnO}_{3}$ heterostructure indicated electron transfer from $\mathrm{Mn}$ to $\mathrm{Ni}$ at the interface [4]. A combined XPS and XAS study of $\mathrm{LaCoO}_{3} / \mathrm{LaTiO}_{3}$ heterostructures also indicated charge transfer to produce $\mathrm{Co}^{2+}$ and $\mathrm{Ti}^{4+}$ valences at the interface [111]. Charge transfer in $\mathrm{SrMnO}_{3} / \mathrm{SrIrO}_{3}$ to produce $\mathrm{Mn}^{3+}$ and $\mathrm{Ir}^{5+}[46,47]$ and $\mathrm{LaMnO}_{3} / \mathrm{SrIrO}_{3}$ to produce $\mathrm{Mn}^{2+}$ and $\mathrm{Ir}^{5}$ + [48] heterostructures has also been demonstrated. Each of these examples agrees with the model put forward by Zhong and Hansmann [104], indicating the value of their approach in predicting interfacial charge transfer and band alignment based on initially aligning the $\mathrm{O} 2 p$ energy bands. Future studies are likely to benefit from moving beyond $3 d$ transition metal systems into the $4 d$ and $5 d$ blocks of the periodic table to generate novel interfacial phenomena.

\section{Experimental design considerations}

We have shown above several examples of the use of in situ XPS studies to probe interfacial phenomena in oxide heterostructures. The availability of an XPS appended to a growth chamber makes these studies very convenient and generates important physical insights as soon as the synthesis is complete. However, the knowledge gleaned from such studies is constrained by both the physical limitations of XPS and by the researcher's chosen design for the synthesized heterostructure. We have already discussed the information depth within the sample for an $\mathrm{Al} \mathrm{K}_{\alpha}$ source, which is limited to $\sim 5 \mathrm{~nm}$ for photoemission normal to the film surface. Thus, band alignment and charge transfer studies are impractical for interfaces that are more than $5 \mathrm{~nm}$ below the surface.

One must also consider which interfaces in a multilayer will exhibit emergent properties, the chemical stability of the 
surface in vacuum and in atmosphere, and potential interference due to overlapping core-level peak energies. In the latter case, $\mathrm{LaNiO}_{3}$ is a good example of a material that cannot be easily examined via XPS due to the overlap of the La $3 d_{3 / 2}$ and the $\mathrm{Ni} 2 p_{3 / 2}$. The $\mathrm{LaNiO}_{3} / \mathrm{LaTiO}_{3}$ charge transfer described above would be very difficult to study in XPS, though the use of the Ni $3 p$ peak does provide one way to probe changes in $\mathrm{Ni}$ valence [112]. Furthermore, the depth sensitivity of XPS means that if two interfaces are present in a multilayer, as in the case of the $\mathrm{LaTiO}_{3} / \mathrm{LaFeO}_{3} / \mathrm{LaTiO}_{3}$ trilayer in Fig. 12(a), then the top interface will generate a majority of the signal and any phenomena at the bottom interface may be difficult or impossible to measure. Finally, while in situ measurements protect against changes due to atmospheric exposure, some surfaces may be modified even during the sample cooldown process [32] or will have intrinsic oxygen vacancies [55]. The deposition of a protective capping layer during the growth process that will not induce any chemical changes in the underlying materials is thus valuable even for in situ studies if a surface is unstable.

\section{Future directions for in situ and related studies}

\section{Hard X-ray photoelectron spectroscopy}

Advances in spectroscopy over the past decade have largely been driven by the development of new techniques from synchrotron light sources. These have included the emergence of hard X-ray photoelectron spectroscopy (HAXPES). A variety of new HAXPES beamlines [113, 114, 115, 116] have been constructed as new light sources are brought online, enabling XPS studies that are not limited by the surface sensitivity of lab sources. Performing XPS studies with X-ray photon energies $>2 \mathrm{keV}$ provides more bulk sensitivity and enables access to transition metal $1 s$ core levels that cannot be examined in a conventional $\mathrm{Al} \mathrm{K}_{\alpha}$ laboratory source. As discussed previously, the IMFP for photoelectrons, $\lambda$, scales with the square root of electron kinetic energy for high kinetic energies. This means that electrons with kinetic energies $\sim 10 \mathrm{keV}$ will have IMFP approaching $10 \mathrm{~nm}$. An IMFP of $10 \mathrm{~nm}$ produces depth sensitivities such that only half of the escaping photoelectrons will be from the top $6.5 \mathrm{~nm}$ of the sample and only $10 \%$ of the signal comes from the surface (top $1.0 \mathrm{~nm}$ ). Conversely, for an IMFP of $1.5 \mathrm{~nm}$ that would be typical for a lab source, half of the signal is generated from the top $1.0 \mathrm{~nm}$ and only $3 \%$ of the signal comes from $5.0 \mathrm{~nm}$ below the surface. Thus, HAXPES measurements enable studies of deeply buried interfaces more than $5 \mathrm{~nm}$ below the film surface that would be impossible with a lab source. Numerous synchrotron studies have made great use of HAXPES to probe oxide interfaces, including the $\mathrm{LaAlO}_{3} / \mathrm{SrTiO}_{3}$ interface $[91,117]$ and the
$\mathrm{LaNiO}_{3}$ surface [53] that we have already discussed. We refer the reader to several good reviews and examples of HAXPES studies, $[118,119,120,121]$ and will focus our attention in this section on the use of the technique to probe $\mathrm{BaSnO}_{3}$.

$\mathrm{BaSnO}_{3}$ is an exciting wide band gap oxide that has been shown to exhibit extremely high electron mobilities at room temperature $\left(150-200 \mathrm{~cm}^{2} / \mathrm{V}\right.$-s) when grown by molecular beam epitaxy [122, 123, 124]. Unlike other perovskite oxides, the conduction band of $\mathrm{BaSnO}_{3}$ is derived from $\mathrm{Sn} 5 s$ orbitals, $[125,126]$ which enable a much greater degree of electronic dispersion and carrier mobility than, i.e., $3 d$ orbitals in $\mathrm{SrTiO}_{3}$. While this dramatic enhancement of mobility is of great benefit for potential future device applications, such as field-effect transistors [127, 128, 129], it also poses challenges to effective doping, both through the use of $n$-type donors [130] and across interfaces through modulation doping and charge transfer [103]. HAXPES has been particularly useful to elucidate the nature of the electronic transport in $\mathrm{BaSnO}_{3}$ and in examining interfacial carrier profiles in stannate heterostructures.

Initial studies of La-doped $\mathrm{BaSnO}_{3}$ via HAXPES focused on understanding the nature of the conduction band profile in the material, by probing electronic states near the Fermi level [125, 126]. Through ex situ synchrotron HAXPES studies with $4 \mathrm{keV}$ photon energy, the first evidence of mobile Sn $5 s$ electrons near the Fermi level was reported [125]. Laboratory-source $\mathrm{Al} \mathrm{K}_{\alpha}$ studies of the same samples could not confirm the presence of carriers at the Fermi edge. The authors attributed this observation to the greater photoelectron cross section for $\mathrm{Sn} 5 s$ with $4 \mathrm{keV}$ photons in comparison to the $1487 \mathrm{eV}$ lab source. However, given continued studies of the stability of dopants in $\mathrm{BaSnO}_{3}$ [130], it also seems possible that atmospheric exposure led to the depletion of the surface carriers, which would explain the absence of observed carriers in the lab-source experiments. Carriers in identically doped $\mathrm{BaSnO}_{3}$ were later observed in a lab-source experiment [131], lending further credence to this possibility. It is also possible that the HAXPES experiments [125] were successful due to the greater probe depth for hard X-rays, which overcomes the surface depletion. This explanation would provide another example of the effects of atmospheric exposure on metastable electronic states near the Fermi level. Further HAXPES studies of $\mathrm{BaSnO}_{3}$ doped with differing La concentrations helped to explain the nature of the conduction band filling and band gap renormalization due to the Burstein-Moss effect [126]. These studies have paved the way for the development of stannate thin films and heterostructures, as research moves from understanding the nature of electronic conduction to confinement of carriers in a high-mobility 2DEG for device applications.

Modulation doping of $\mathrm{BaSnO}_{3}$ through engineering of band alignment and charge transfer is a key requirement for 
(a)

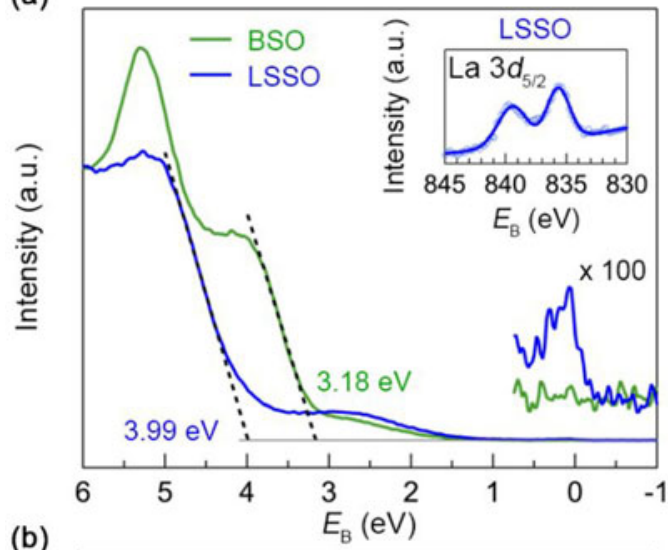

(b)

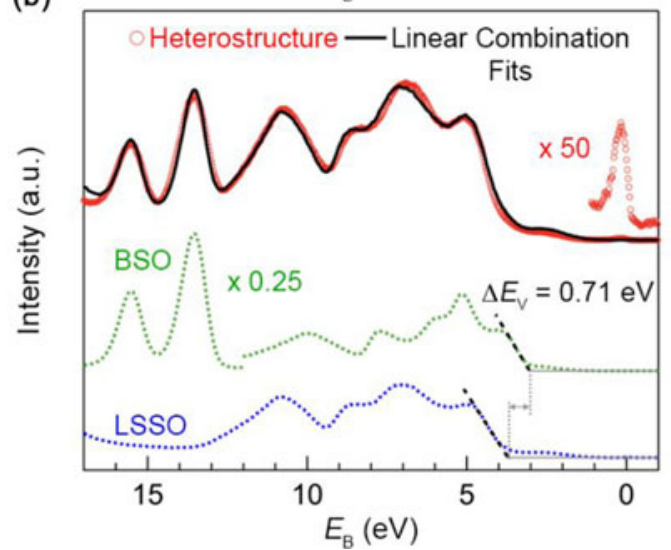

(c)

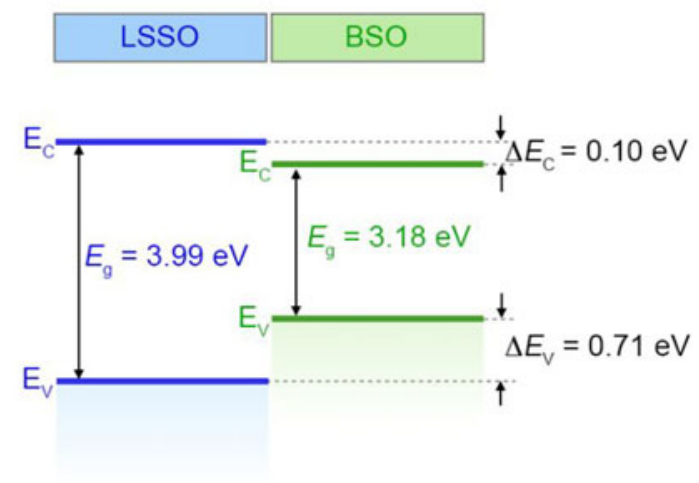

(d)

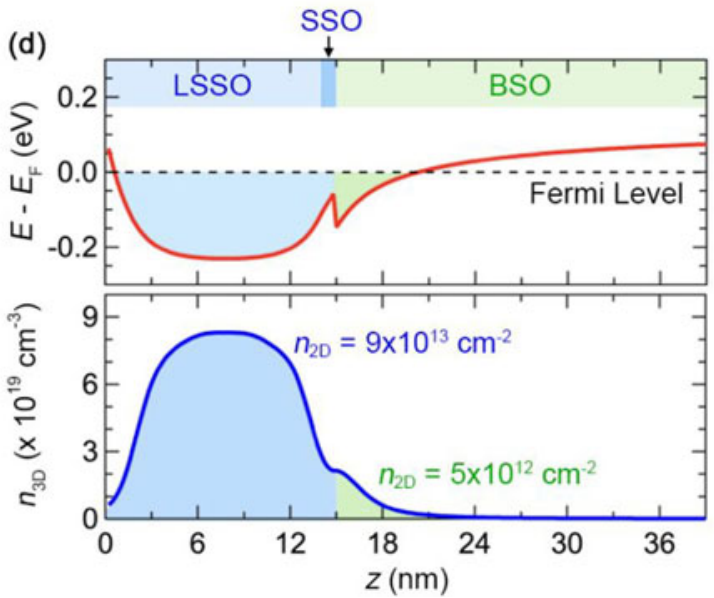

Figure 13: Band alignment at La-doped $\left.\mathrm{SrSnO}_{3}(\mathrm{LSSO}) / \mathrm{SrSnO}_{3} \mathrm{SSO}\right) / \mathrm{BaSnO}_{3}$ (BSO) interface. (a) VB spectra of the reference $\mathrm{BSO}$ (green) (56 $\mathrm{nm} \mathrm{BSO} / \mathrm{SrTiO}_{3}(001)$ ) and LSSO (blue) $\left(41 \mathrm{~nm} \mathrm{LSSO} / 8 \mathrm{~nm} \mathrm{SSO} / \mathrm{GdSCO}_{3}(110)\right)$ films. Electronic states near the Fermi states are magnified. Inset shows the La $3 d_{5 / 2}$ core-level X-ray photoelectron spectra, (b) VB spectra of the SSO/BSO heterostructure (red) along with the fit (black) using linear combination of the reference VB spectra (dotted green and blue lines) to determine the VB offset. (c) An energy level flat-band diagram showing the measured band offsets between LSSO and BSO, and (d) conduction band minima (red) referenced to the Fermi level (top panel) and 3D carrier density, $n_{3 \mathrm{D}}$ (blue) as a function of depth for the SSO/BSO (bottom panel). The shaded regions indicate 2D density in LSSO and BSO layers after the charge transfer. Reprinted with permission from A. Prakash et al. [132]. Copyright 2019 American Chemical Society.

a 2DEG structure. Impurity scattering from La donor ions is a key limitation to the further enhancements of mobility in electron-doped $\mathrm{BaSnO}_{3}$ [123]. To overcome this, engineering of a heterostructure with conduction band electrons at greater energies than the $\mathrm{BaSnO}_{3}$ conduction band is necessary. In one such heterostructure, a $14 \mathrm{~nm}$ La-doped $\mathrm{SrSnO}_{3}$ top layer was engineered such that free carriers in that layer could flow "downhill" to an undoped $\mathrm{BaSnO}_{3}$ below the $\mathrm{SrSnO}_{3}$ layer to form a 2DEG [132]. Given the thickness of the $\mathrm{SrSnO}_{3}$ top layer, synchrotron HAXPES studies were needed to probe the buried interface. Using a photon energy of $5.93 \mathrm{keV}$, the group was able to extract the interfacial band alignment and estimate the degree of charge transfer into the underlying $\mathrm{BaSnO}_{3}$. These results are shown in Fig. 13. Interestingly, the extracted band profile in Fig. 13(d) could be explained by surface carrier depletion from atmospheric exposure, as discussed above regarding La-doped $\mathrm{BaSnO}_{3}$ [125]. These results show that the band alignment techniques that have been used for in situ studies for many years can be applied to more challenging structures using hard X-ray sources that probe more deeply into materials. Continued development of HAXPES will dramatically improve the ability to measure interfacial phenomena in heterostructures that are not limited to a few $\mathrm{nm}$ in thickness due to the lab-based X-ray energies.

\section{Standing-wave XPS}

While HAXPES measurements are one approach to probe more deeply within a material and examine both bulk electronic structure and buried interfaces, the underlying physics of the photoemission process is unchanged. As we showed above, HAXPES measurements can still be affected by surface chemistry of the films. The standing-wave XPS (SW-XPS) approach has emerged over the past decade, specifically focusing on studies of superlattice structures that exhibit regularly repeating interfaces. By varying the incoming X-ray angle such that the Bragg diffraction condition is satisfied for the superlattice, the intensity of the electric field is modulated 

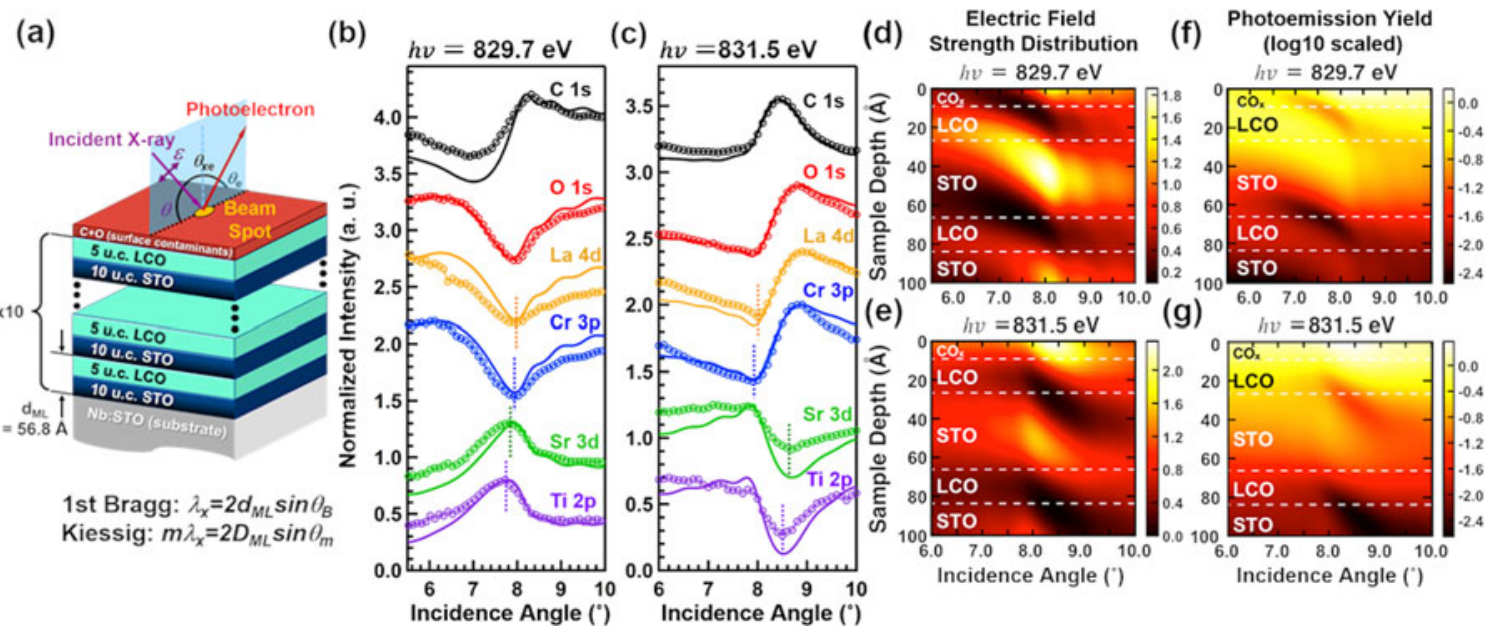

$h v=831.5 \mathrm{eV}$

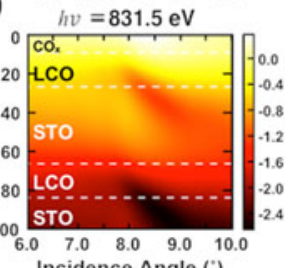

Figure 14: (a) Schematic of the superlattice made up of 10 bilayers of LCO and STO, consisting of 5 UCs of LCO, $17.6 \AA$ thick, and 10 UCs of STO, $39.2 \AA$ thick, grown epitaxially on a $\mathrm{Nb}$-doped STO(001) substrate. The two sources of the standing-wave structure in the rocking curves are indicated: Bragg reflection from the multilayer with period $d_{\mathrm{ML}}$ and Kiessig fringes associated with the full thickness of the multilayer stack $D_{\mathrm{ML}}$. Experimental (open circles) and simulated (solid) rocking curves of representative elemental states at photon energies of (b) $829.7 \mathrm{eV}$ and (c) $831.5 \mathrm{eV}$. The colored dash lines are the guides to the eye to indicate the phase of the rocking curves in ( $b$ and $c$ ) to show sensitivity to the interfacial termination $\left(\mathrm{SrO} / \mathrm{CrO}_{2}\right.$ versus $\left.\mathrm{TiO}_{2} / \mathrm{LaO}\right)$. The electric field strength distribution derived from X-ray optics calculations at two energies near the La $M_{5}$ resonance, (d) $829.7 \mathrm{eV}$ and (e) $831.5 \mathrm{eV}$ as a function of sample depth and incidence angle. Note the significant shift in position between the two energies. The corresponding photoemission yields, ( $\mathrm{f}$ and $\mathrm{g}$ ), plotted on log 10 scales. Adapted figure with permission from S.-C. Lin et al. [136] . Copyright 2018 by the American Physical Society.

across the interface. A stronger Bragg reflection produces a greater standing-wave intensity and greater modulation of the electric field strength across the repeating superlattice formula unit. The physics of SW-XPS has been described in more detail in various references $[118,133,134,135]$. Here, we aim to discuss the applications of SW-XPS to understanding interfacial phenomena and present opportunities to expand on this unique approach by designing interfacial materials that will be well suited for SW-XPS studies.

As an initial example, we present a study of layer-resolved band alignment in a superlattice achieved through careful design of a superlattice structure. The structure and electric field strength modulation is shown schematically in Fig. 14 for a study of a $\mathrm{LaCrO}_{3}-\mathrm{SrTiO}_{3}$ superlattice using $\sim 830 \mathrm{eV}$ photon energy near the La $M_{5}$ resonance. [136] In this case, the formula of $10 \mathrm{UCs}$ of $\mathrm{SrTiO}_{3}$ followed by $5 \mathrm{UCs}$ of $\mathrm{LaCrO}_{3}$ over 10 repeating layers in the superlattice was chosen to maximize the Bragg reflection and thus the sensitivity to individual interfaces. By choosing the superlattice periodicity prior to growth and considering the subsequent SW-XPS experiment, the largest standing-wave response ever achieved was reported, with individual core-level intensities varying by $\sim 50 \%$ as the X-ray angle was varied across the Bragg peak. This enabled the extraction of the band alignment across the entire 15-unit-cell structure through careful modeling of the core-level peak positions as a function of incoming angle, producing good agreement with DFT models [136, 137].

The SW-XPS technique has been used to probe changes in interfacial chemistry in a variety of other superlattice systems.
Evidence for $\mathrm{Ni}^{2+}$ valence near the interface with $\mathrm{SrTiO}_{3}$ in 4 u.c. $\mathrm{LaNiO}_{3}-4$ u.c. $\mathrm{SrTiO}_{3}$ superlattices was reported [138]. Studies of $\mathrm{LaNiO}_{3}-\mathrm{CaMnO}_{3}$ superlattices showed similar effects, with $\mathrm{Ni}^{2+}$ states observed at the interface [139]. Likewise, changes in the interfacial $\mathrm{Mn}$ valence in ( $\mathrm{La}, \mathrm{Sr}$ ) $\mathrm{MnO}_{3}-\mathrm{SrTiO}_{3}$ superlattices were also observed [140]. Collectively, these studies have shown that the technique provides additional sensitivity to changing interfacial chemical states at interfaces. The X-ray standing wave provides a novel way to isolate the interfacial contribution to the detected signal that goes beyond conventional angle-resolved XPS. However, the design of the superlattice and choice of materials system under examination plays a significant role in how much information can be extracted from the measurement. For example, superlattices with large differences in density between layers produce larger superlattice Bragg peaks, so that a superlattice comprised of, i.e., $\mathrm{SrTiO}_{3}$ and $\mathrm{LaTiO}_{3}$ layers will produce a greater peak than one comprised of, i.e., $\mathrm{SrTiO}_{3}$ and $\mathrm{SrVO}_{3}$ or $\mathrm{LaTiO}_{3}$ and $\mathrm{LaVO}_{3}$ layers. This means that the technique is currently only suitable for select combinations of materials.

Ideally, SW-XPS could be used to measure not simply changes in interfacial chemistry but also to probe emergent interfacial electronic states near the Fermi level. There have also been some efforts to employ SW-XPS to generate ARPES information as a function of depth within the material, including studies of $\mathrm{SrTiO}_{3}-\mathrm{GdTiO}_{3}$ [141] and $\mathrm{SrTiO}_{3}-(\mathrm{La}, \mathrm{Sr})$ $\mathrm{MnO}_{3}$ [142] superlattices. These results are promising demonstrations of the technique but provided limited insights into emergent interfacial physical phenomena due in large part to 
the relatively weak X-ray standing wave. The weakness of the standing-wave can be observed in small variation of the corelevel peak intensities as a function of angle $(\sim 20-30 \%)$. This means that differences in the ARPES data as a function of angle are fairly small and it is difficult to extract clear information about the electronic structure in individual layers of the superlattice or at a particular interface. In most cases of SW-XPS studies to date, the challenge has not been the technique but the limitation of the samples themselves. We suggest that a somewhat different model could bear fruit.

Because the photon energies for most SW-XPS studies have been in the soft X-ray regime where it is possible to take advantage of elemental resonances to enhance reflectivity, the measurements are still limited to the top few nm of the sample. Thus, the underlying superlattice serves only to generate the $\mathrm{X}$-ray standing wave that modulates the field strength across the topmost repeat layer of the sample and does not have a meaningful effect on the actual data being measured. It, therefore, makes sense to choose an underlying superlattice solely from the perspective of generating the strongest standing-wave intensity while not confusing the data interpretation from the relevant interface in other ways. In this sense, the superlattice serves as a novel substrate for the actual experimental interface, as has been applied in other cases for non-epitaxial films such as the $\mathrm{Fe}-\mathrm{MgO}$ interface [143] and ambient-pressure XPS measurements on $\mathrm{Fe}_{2} \mathrm{O}_{3}$ [144]. For example, a $\mathrm{SrTiO}_{3}-\mathrm{BaTiO}_{3}$ superlattice is relatively easy to synthesize and would provide strong standing-wave effects across the $\mathrm{Ba} M_{5}$ resonance $(\sim 780 \mathrm{eV})$. Predictions of superlattice Bragg peaks for various $\mathrm{BTO}_{n}-\mathrm{STO}_{n}$ superlattice structures using the X-ray Interactions with Matter website $[145,146]$ provided by the Center for X-ray Optics at Lawrence Berkeley National Laboratory are shown in Fig. 15(a). The electric field strength varies as the square root of reflectivity, meaning that values greater than 0.1 on the scale (dotted line) will produce modulations of XPS peak intensity of $30 \%$ or better. The superlattices could then be used as X-ray mirror substrates for any number of intriguing interfacial materials to produce much stronger standing-wave effects. A schematic of such a heterostructure is shown in Fig. $15(\mathrm{~b})$, with a $\mathrm{BTO}_{5}-\mathrm{STO}_{5}$ superlattice with an arbitrary $\mathrm{SrMO}_{3}-\mathrm{SrM}^{\prime} \mathrm{O}_{3}$ interface highlighted in yellow that will be strongly probed by the standing wave from the underlying mirror. Given this opportunity to shift the approach to standing-wave measurements, groups should consider the SW-XPS experiment when designing both their superlattice mirror layer and the interface of interest. Others have proposed additional approaches to enhance standing-wave effects in these measurements [133]. Ideally, collaborations between synthesis groups and experts in SW-XPS early in the research process will produce samples that are particularly well suited to answer specific questions about the properties of an interface.
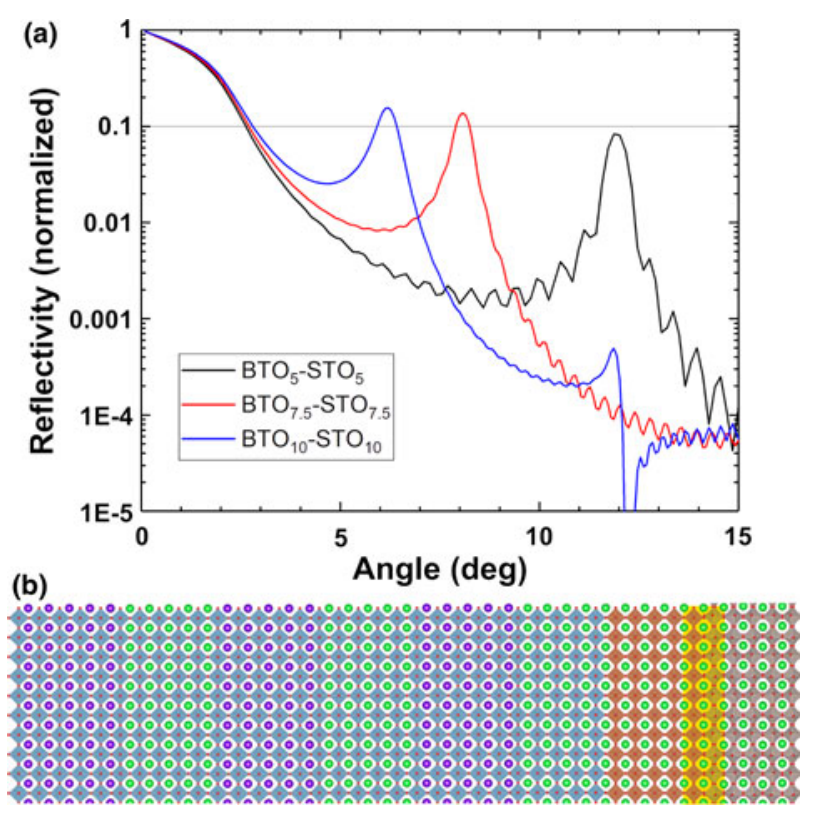

Figure 15: (a) Modeled X-ray reflectivity for three $n$-unit cell $\mathrm{BaTiO}_{3}\left(\mathrm{BTO}_{n}\right)$ / $n$-unit cell $\mathrm{SrTiO}_{3}\left(\mathrm{STO}_{n}\right)$ superlattices on $\mathrm{SrTiO}_{3}$ substrates at the $\mathrm{Ba} \mathrm{M}_{5}$ energy resonance; (b) Schematic of $\mathrm{BTO}_{5}-\mathrm{STO}_{5}$ superlattice mirror to probe an arbitrary $\mathrm{SrBO}_{3}-\mathrm{SrB}^{\prime} \mathrm{O}_{3}$ interface (highlighted in yellow).

\section{Future applications}

In summary, synchrotron XPS techniques have generated significant new insights into buried oxide interfaces by taking advantage of the variable $\mathrm{X}$-ray energies that are provided from a synchrotron source that cannot be replicated in a lab. Many of these approaches have only been pursued over the past decade, however, leaving significant opportunities to refine the practices to better extract novel physics from the experiments. For example, many beamlines have begun to integrate MBE or PLD growth systems in situ as has been done in a lab environment for many years $[147,148]$. We have shown in this review that surface exposure must be considered when interpreting results, even for HAXPES studies. It thus makes sense to perform SW-XPS and HAXPES studies using in situ growth capabilities at the beamline whenever possible. Alternatively, the use of a vacuum suitcase to transport samples from a home synthesis laboratory to the user facility can enable synchrotron studies that replicate in situ conditions.

Another promising new opportunity has been enabled by the development of lab-based HAXPES instruments [149]. Rather than traditional $\mathrm{Al}$ or $\mathrm{Mg}$ sources, these commercially available instruments employ a $3 d$ transition metal or metalloid such as $\mathrm{Cr}$ or $\mathrm{Ga}$ to produce monochromatic $\mathrm{K} \alpha$ photons with energies greater than $5 \mathrm{keV}$. The recent development of a liquid-metal Ga source with a Rowland circle monochromator has produced fluxes that are within a factor of 200 of synchrotron sources, making it possible to perform measurements in 
reasonable times in a lab environment [149]. To date, we know of no laboratory that has an oxide or other complex materials film growth chamber attached in situ to a HAXPES instrument, but this would be an excellent resource for future film synthesis user facilities. An instrument suite integrating film synthesis and the large probing depth of a hard X-ray source in situ would be an invaluable resource for studies of charge transfer and band alignment in future complex heterostructures like the one presented in Fig. 13 [132].

\section{Summary}

In conclusion, we have examined the state-of-the-art in oxide thin films and interfaces by focusing on the synergy that is possible when epitaxial film synthesis and XPS are integrated in situ. We have shown how XPS can be used to improve the synthesis process to observe film contamination, stoichiometry, surface termination, and surface chemistry. These day-to-day measurements can be extremely valuable to film growers as they optimize the growth process. By demonstrating the effects that metastable surfaces and atmospheric exposure can have on measured film properties, we show both the benefit of performing such measurements in situ. In general, energetically unfavorable states will not be preserved at a film surface. These include the reduction of $\mathrm{Ni}^{3+}$ to $\mathrm{Ni}^{2+}$ and the oxidation of $\mathrm{Ti}^{3+}$ to $\mathrm{Ti}^{4+}$ and $\mathrm{Sn}^{3+}$ to $\mathrm{Sn}^{4+}$ upon atmospheric exposure. A corollary to these effects is the care that must be taken in interpreting other spectroscopy results as well as other measurements such as electronic transport.

We have also shown how more complex XPS experiments can be designed to measure interfacial intermixing, valence band offsets, and charge transfer. By examining several case studies, we showed the importance of designing thin film heterostructures with the subsequent XPS experiment in mind. Predictive models for band alignment and interfacial charge transfer based on the alignment of the $\mathrm{O} 2 p$ bands can be valuable in selecting interfaces that are likely to generate novel properties, which are then readily measured via in situ XPS. Finally, we presented future directions for XPS-based studies of oxide interfaces that leverage advances in synchrotron and lab-based technologies to achieve high impact results. Bridging the gap between synthesis and characterization in these future studies will hopefully provide the oxide thin film community with a better understanding of the complex chemical and physical phenomena that drive emergent behavior in oxide heterostructures.

\section{Acknowledgments}

We thank Shalinee Chikara for assistance with the $\mathrm{LaCoO}_{3}$ deposition and XPS data acquisition shown in the paper. The authors gratefully acknowledge support from the Air Force Office of Scientific Research under award number FA955020-1-0034 and from the National Science Foundation Division of Materials Research under award number NSF-DMR-1809847. M.D.B. acknowledges fellowship support through the Alabama EPSCOR Graduate Research Scholars Program.

\section{References}

1. A. Ohtomo and H.Y. Hwang: A high-mobility electron gas at the $\mathrm{LaAlO}_{3} / \mathrm{SrTiO}_{3}$ heterointerface. Nature 427, 423 (2004).

2. J.A. Mundy, C.M. Brooks, M.E. Holtz, J.A. Moyer, H. Das, A.F. Rébola, J.T. Heron, J.D. Clarkson, S.M. Disseler, Z. Liu, A. Farhan, R. Held, R. Hovden, E. Padgett, Q. Mao, H. Paik, R. Misra, L.F. Kourkoutis, E. Arenholz, A. Scholl,

J.A. Borchers, W.D. Ratcliff, R. Ramesh, C.J. Fennie, P. Schiffer, D.A. Muller, and D.G. Schlom: Atomically engineered ferroic layers yield a room-temperature magnetoelectric multiferroic. Nature 537, 523 (2016).

3. D. Yi, P. Yu, Y.-C. Chen, H.-H. Lee, Q. He, Y.-H. Chu, and R. Ramesh: Tailoring magnetoelectric coupling in $\mathrm{BiFeO}_{3} /$ $\mathrm{La}_{0.7} \mathrm{Sr}_{0.3} \mathrm{MnO}_{3}$ heterostructure through the interface engineering. Adv. Mater. 31, 1806335 (2019).

4. J. Hoffman, I.C. Tung, B.B. Nelson-Cheeseman, M. Liu, J.W. Freeland, and A. Bhattacharya: Charge transfer and interfacial magnetism in $\left(\mathrm{LaNiO}_{3}\right)_{\mathrm{n}} /\left(\mathrm{LaMnO}_{3}\right)_{2}$ superlattices. Phys. Rev. B 88, 144411 (2013).

5. A.S. Disa, D.P. Kumah, A. Malashevich, H. Chen, D.A. Arena, E.D. Specht, S. Ismail-Beigi, F.J. Walker, and C.H. Ahn: Orbital engineering in symmetry-breaking polar heterostructures. Phys. Rev. Lett. 114, 026801 (2015).

6. M. Coll, J. Fontcuberta, M. Althammer, M. Bibes, H. Boschker, A. Calleja, G. Cheng, M. Cuoco, R. Dittmann, B. Dkhil, I. El Baggari, M. Fanciulli, I. Fina, E. Fortunato, C. Frontera, S. Fujita, V. Garcia, S.T.B. Goennenwein, C.-G. Granqvist, J. Grollier, R. Gross, A. Hagfeldt, G. Herranz, K. Hono, E. Houwman, M. Huijben, A. Kalaboukhov, D.J. Keeble, G. Koster, L.F. Kourkoutis, J. Levy, M. Lira-Cantu, J.L. MacManus-Driscoll, J. Mannhart, R. Martins, S. Menzel, T. Mikolajick, M. Napari, M.D. Nguyen, G. Niklasson, C. Paillard, S. Panigrahi, G. Rijnders, F. Sánchez, P. Sanchis, S. Sanna, D.G. Schlom, U. Schroeder, K.M. Shen, A. Siemon, M. Spreitzer, H. Sukegawa, R. Tamayo, J. van den Brink, N. Pryds, and F.M. Granozio: Towards oxide electronics: A roadmap. Appl. Surf. Sci. 482, 1 (2019).

7. M. Uchida and M. Kawasaki: Topological properties and functionalities in oxide thin films and interfaces. J. Phys. D: Appl. Phys. 51, 143001 (2018). 
8. Z. Huang, Ariando, X.R. Wang, A. Rusydi, J. Chen, H. Yang, and T. Venkatesan: Interface engineering and emergent phenomena in oxide heterostructures. Adv. Mater. 30, 1802439 (2018)

9. M. Brahlek, A.S. Gupta, J. Lapano, J. Roth, H.-T. Zhang, L. Zhang, R. Haislmaier, and R. Engel-Herbert: Frontiers in the growth of complex oxide thin films: Past, present, and future of hybrid MBE. Adv. Funct. Mater. 28, 1702772 (2018).

10. Y.E. Suyolcu, G. Christiani, P.A. van Aken, and G. Logvenov: Design of complex oxide interfaces by oxide molecular beam epitaxy. J Supercond. Nov. Magn. 33, 107 (2020).

11. D.G. Schlom: Perspective: Oxide molecular-beam epitaxy rocks!. APL Mater. 3, 062403 (2015)

12. R. Groenen, J. Smit, K. Orsel, A. Vailionis, B. Bastiaens, M. Huijben, K. Boller, G. Rijnders, and G. Koster: Research Update: Stoichiometry controlled oxide thin film growth by pulsed laser deposition. APL Mater. 3, 070701 (2015).

13. H.N. Lee, S.S. Ambrose Seo, W.S. Choi, and C.M. Rouleau: Growth control of oxygen stoichiometry in homoepitaxial $\mathrm{SrTiO}_{3}$ films by pulsed laser epitaxy in high vacuum. Sci. Rep. 6, 19941 (2016).

14. H. Nakamura and T. Kimura: Electric field tuning of spin-orbit coupling in $\mathrm{KTaO}_{3}$ field-effect transistors. Phys. Rev. B 80, 121308 (2009)

15. G. Herranz, M. Basletić, M. Bibes, C. Carrétéro, E. Tafra, E. Jacquet, K. Bouzehouane, C. Deranlot, A. Hamzić, J.-M. Broto, A. Barthélémy, and A. Fert: High mobility in $\mathrm{LaAlO}_{3} / \mathrm{SrTiO}_{3}$ heterostructures: Origin, dimensionality, and perspectives. Phys. Rev. Lett. 98, 216803 (2007).

16. M. Hosoda, Y. Hikita, H.Y. Hwang, and C. Bell: Transistor operation and mobility enhancement in top-gated $\mathrm{LaAlO}_{3} /$ $\mathrm{SrTiO}_{3}$ heterostructures. Appl. Phys. Lett. 103, 103507 (2013).

17. H. Liang, L. Cheng, L. Wei, Z. Luo, G. Yu, C. Zeng, and Z. Zhang: Nonmonotonically tunable Rashba spin-orbit coupling by multiple-band filling control in $\mathrm{SrTiO}_{3}$-based interfacial d-electron gases. Phys. Rev. B 92, 075309 (2015).

18. E. Lesne, Y. Fu, S. Oyarzun, J.C. Rojas-Sánchez, D.C. Vaz, H. Naganuma, G. Sicoli, J.-P. Attané, M. Jamet, E. Jacquet, J.-M. George, A. Barthélémy, H. Jaffrès, A. Fert, M. Bibes, and L. Vila: Highly efficient and tunable spin-to-charge conversion through Rashba coupling at oxide interfaces. Nat. Mater. 15, 1261 (2016).

19. S.A. Chambers, M.H. Engelhard, V. Shutthanandan, Z. Zhu, T.C. Droubay, L. Qiao, P.V. Sushko, T. Feng, H.D. Lee, T. Gustafsson, E. Garfunkel, A.B. Shah, J.-M. Zuo, and Q.M. Ramasse: Instability, intermixing and electronic structure at the epitaxial heterojunction. Surf. Sci. Rep. 65, 317 (2010).

20. M.P. Warusawithana, C. Richter, J.A. Mundy, P. Roy, J. Ludwig, S. Paetel, T. Heeg, A.A. Pawlicki, L.F. Kourkoutis, M. Zheng, M. Lee, B. Mulcahy, W. Zander, Y. Zhu, J. Schubert,
J.N. Eckstein, D.A. Muller, C.S. Hellberg, J. Mannhart, and D.G. Schlom: $\mathrm{LaAlO}_{3}$ stoichiometry is key to electron liquid formation at $\mathrm{LaAlO}_{3} / \mathrm{SrTiO}_{3}$ interfaces. Nat. Commun. 4, 2351 (2013).

21. E. Breckenfeld, N. Bronn, J. Karthik, A.R. Damodaran, S. Lee, N. Mason, and L.W. Martin: Effect of growth induced (non) stoichiometry on interfacial conductance in $\mathrm{LaAlO}_{3} / \mathrm{SrTiO}_{3}$. Phys. Rev. Lett. 110, 196804 (2013).

22. Y. Segal, J.H. Ngai, J.W. Reiner, F.J. Walker, and C.H. Ahn: $\mathrm{X}$-ray photoemission studies of the metal-insulator transition in $\mathrm{LaAlO}_{3} / \mathrm{SrTiO}_{3}$ structures grown by molecular beam epitaxy. Phys. Rev. B 80, 241107 (2009).

23. S.A. Chambers: Understanding the mechanism of conductivity at the $\mathrm{LaAlO}_{3} / \mathrm{SrTiO}_{3}(001)$ interface. Surf. Sci. 605, 1133 (2011).

24. P. Xu, T.C. Droubay, J.S. Jeong, K. Andre Mkhoyan, P.V. Sushko, S.A. Chambers, and B. Jalan: Quasi 2D ultrahigh carrier density in a complex oxide broken-gap heterojunction. Adv. Mater. Interfaces 3, 1500432 (2015).

25. P. Xu, Y. Ayino, C. Cheng, V.S. Pribiag, R.B. Comes,

P.V. Sushko, S.A. Chambers, and B. Jalan: Predictive control over charge density in the two-dimensional electron gas at the polar-nonpolar $\mathrm{NdTiO}_{3} / \mathrm{SrTiO}_{3}$ interface. Phys. Rev. Lett. 117, 106803 (2016).

26. P. Moetakef, T.A. Cain, D.G. Ouellette, J.Y. Zhang, D.O. Klenov, A. Janotti, C.G. Van de Walle, S. Rajan, S.J. Allen, and S. Stemmer: Electrostatic carrier doping of $\mathrm{GdTiO}_{3} / \mathrm{SrTiO}_{3}$ interfaces. Appl. Phys. Lett. 99, 232116 (2011).

27. J.S. Kim, S.S.A. Seo, M.F. Chisholm, R.K. Kremer,

H.-U. Habermeier, B. Keimer, and H.N. Lee: Nonlinear Hall effect and multichannel conduction in $\mathrm{LaTiO}_{3} / \mathrm{SrTiO}_{3}$ superlattices. Phys. Rev. B 82, 201407 (2010).

28. J. Biscaras, N. Bergeal, S. Hurand, C. Grossetête, A. Rastogi, R.C. Budhani, D. LeBoeuf, C. Proust, and J. Lesueur: Two-dimensional superconducting phase in $\mathrm{LaTiO}_{3} / \mathrm{SrTiO}_{3}$ heterostructures induced by high-mobility carrier doping. Phys. Rev. Lett. 108, 247004 (2012).

29. P. Moetakef, J.Y. Zhang, A. Kozhanov, B. Jalan, R. Seshadri, S.J. Allen, and S. Stemmer: Transport in ferromagnetic $\mathrm{GdTiO}_{3} / \mathrm{SrTiO}_{3}$ heterostructures. Appl. Phys. Lett. 98, 112110 (2011).

30. Y. Ayino, P. Xu, J. Tigre-Lazo, J. Yue, B. Jalan, and V.S. Pribiag: Ferromagnetism and spin-dependent transport at a complex oxide interface. Phys. Rev. Mater. 2, 031401 (2018).

31. P. Scheiderer, M. Schmitt, J. Gabel, M. Zapf, M. Stübinger, P. Schütz, L. Dudy, C. Schlueter, T.-L. Lee, M. Sing, and R. Claessen: Tailoring materials for Mottronics: Excess oxygen doping of a prototypical Mott insulator. Adv. Mater. 30, 1706708 (2018).

32. R. Aeschlimann, D. Preziosi, P. Scheiderer, M. Sing, S. Valencia, J. Santamaria, C. Luo, H. Ryll, F. Radu, 
R. Claessen, C. Piamonteze, and M. Bibes: A living-dead magnetic layer at the surface of ferrimagnetic $\mathrm{DyTiO}_{3}$ thin films. Adv. Mater. 30, 1707489 (2018).

33. P.D.C. King, R.H. He, T. Eknapakul, P. Buaphet, S.-K. Mo, Y. Kaneko, S. Harashima, Y. Hikita, M.S. Bahramy, C. Bell, Z. Hussain, Y. Tokura, Z.-X. Shen, H.Y. Hwang,

F. Baumberger, and W. Meevasana: Subband structure of a twodimensional electron gas formed at the polar surface of the strong spin-orbit perovskite $\mathrm{KTaO}_{3}$. Phys. Rev. Lett. 108, 117602 (2012).

34. A.F. Santander-Syro, C. Bareille, F. Fortuna, O. Copie,

M. Gabay, F. Bertran, A. Taleb-Ibrahimi, P. Le Fèvre,

G. Herranz, N. Reyren, M. Bibes, A. Barthélémy, P. Lecoeur,

J. Guevara, and M.J. Rozenberg: Orbital symmetry reconstruction and strong mass renormalization in the two-dimensional electron gas at the surface of $\mathrm{KTaO}_{3}$. Phys. Rev. B 86, 121107 (2012).

35. J. Thompson, J. Hwang, J. Nichols, J.G. Connell, S. Stemmer, and S.S.A. Seo: Alleviating polarity-conflict at the heterointerfaces of $\mathrm{KTaO}_{3} / \mathrm{GdScO}_{3}$ polar complex-oxides. Appl. Phys. Lett. 105, 102901 (2014).

36. H. Zhang, X. Yan, X. Zhang, S. Wang, C. Xiong, H. Zhang, S. Qi, J. Zhang, F. Han, N. Wu, B. Liu, Y. Chen, B. Shen, and J. Sun: Unusual electric and optical tuning of $\mathrm{KTaO}_{3}$-based two-dimensional electron gases with $5 \mathrm{~d}$ orbitals. ACS Nano 13, 609 (2019).

37. H. Zhang, H. Zhang, X. Yan, X. Zhang, Q. Zhang, J. Zhang, F. Han, L. Gu, B. Liu, Y. Chen, B. Shen, and J. Sun: Highly mobile two-dimensional electron gases with a strong gating effect at the amorphous $\mathrm{LaAlO}_{3} / \mathrm{KTaO}_{3}$ interface. ACS Appl. Mater. Interfaces 9, 36456 (2017).

38. M. Setvin, M. Reticcioli, F. Poelzleitner, J. Hulva, M. Schmid, L.A. Boatner, C. Franchini, and U. Diebold: Polarity compensation mechanisms on the perovskite surface $\mathrm{KTaO}_{3}(001)$. Science 359, 572 (2018).

39. B.J. Kim, H. Jin, S.J. Moon, J.-Y. Kim, B.-G. Park, C.S. Leem, J. Yu, T.W. Noh, C. Kim, S.-J. Oh, J.-H. Park, V. Durairaj, G. Cao, and E. Rotenberg: Novel $\mathrm{J}_{\mathrm{eff}}=1 / 2$ Mott state induced by relativistic spin-orbit coupling in $\mathrm{Sr}_{2} \mathrm{IrO}_{4}$. Phys. Rev. Lett. 101, 076402 (2008).

40. H.-S. Kim, Y. Chen, and H.-Y. Kee: Surface states of perovskite iridates $\mathrm{AIrO}_{3}$ : Signatures of a topological crystalline metal with nontrivial $\mathrm{Z}_{2}$ index. Phys. Rev. B 91, 235103 (2015).

41. Y.F. Nie, P.D.C. King, C.H. Kim, M. Uchida, H.I. Wei, B.D. Faeth, J.P. Ruf, J.P.C. Ruff, L. Xie, X. Pan, C.J. Fennie, D.G. Schlom, and K.M. Shen: Interplay of spin-orbit interactions, dimensionality, and octahedral rotations in semimetallic $\mathrm{SrIrO}_{3}$. Phys. Rev. Lett. 114, 016401 (2015).

42. P. Schütz, D. Di Sante, L. Dudy, J. Gabel, M. Stübinger, M. Kamp, Y. Huang, M. Capone, M.-A. Husanu, V.N. Strocov,
G. Sangiovanni, M. Sing, and R. Claessen:

Dimensionality-driven metal-insulator transition in spin-orbit-coupled $\mathrm{SrIrO}_{3}$. Phys. Rev. Lett. 119, 256404 (2017).

43. D.J. Groenendijk, C. Autieri, J. Girovsky, M.C. Martinez-Velarte, N. Manca, G. Mattoni, A.M.R.V.L. Monteiro, N. Gauquelin, J. Verbeeck, A.F. Otte, M. Gabay, S. Picozzi, and A.D. Caviglia: Spin-orbit semimetal $\mathrm{SrIrO}_{3}$ in the two-dimensional limit. Phys. Rev. Lett. 119, 256403 (2017).

44. T.J. Anderson, S. Ryu, H. Zhou, L. Xie, J.P. Podkaminer, Y. Ma, J. Irwin, X.Q. Pan, M.S. Rzchowski, and C.B. Eom: Metastable honeycomb $\mathrm{SrTiO}_{3} / \mathrm{SrIrO}_{3}$ heterostructures. Appl. Phys. Lett. 108, 151604 (2016).

45. D. Meyers, Y. Cao, G. Fabbris, N.J. Robinson, L. Hao, C. Frederick, N. Traynor, J. Yang, J. Lin, M.H. Upton, D. Casa, J.-W. Kim, T. Gog, E. Karapetrova, Y. Choi, D. Haskel, P.J. Ryan, L. Horak, X. Liu, J. Liu, and M.P.M. Dean: Magnetism in iridate heterostructures leveraged by structural distortions. Sci. Rep. 9, 4263 (2019).

46. J. Nichols, X. Gao, S. Lee, T.L. Meyer, J.W. Freeland, V. Lauter, D. Yi, J. Liu, D. Haskel, J.R. Petrie, E.-J. Guo, A. Herklotz, D. Lee, T.Z. Ward, G. Eres, M.R. Fitzsimmons, and H.N. Lee: Emerging magnetism and anomalous Hall effect in iridate-manganite heterostructures. Nat. Commun. 7, 12721 (2016).

47. S. Okamoto, J. Nichols, C. Sohn, S.Y. Kim, T.W. Noh, and H.N. Lee: Charge transfer in iridate-manganite superlattices. Nano Lett. 17, 2126 (2017).

48. T.S. Suraj, G.J. Omar, H. Jani, M.M. Juvaid, S. Hooda, A. Chaudhuri, A. Rusydi, K. Sethupathi, T. Venkatesan, A. Ariando, and M.S.R. Rao: Tunable and enhanced rashba spin-orbit coupling in iridate-manganite heterostructures. arXiv:2004.00800 [cond-mat, physics:physics] (2020).

49. Y. Cao, X. Liu, M. Kareev, D. Choudhury, S. Middey, D. Meyers, J.-W. Kim, P.J. Ryan, J.W. Freeland, and J. Chakhalian: Engineered Mott ground state in a $\mathrm{LaTiO}_{3+\delta} /$ $\mathrm{LaNiO}_{3}$ heterostructure. Nat. Commun. 7, 10418 (2016).

50. D.P. Kumah, A.S. Disa, J.H. Ngai, H. Chen, A. Malashevich, J.W. Reiner, S. Ismail-Beigi, F.J. Walker, and C.H. Ahn: Tuning the structure of nickelates to achieve two-dimensional electron conduction. Adv. Mater. 26, 1935 (2014).

51. D.P. Kumah, A. Malashevich, A.S. Disa, D.A. Arena, F.J. Walker, S. Ismail-Beigi, and C.H. Ahn: Effect of surface termination on the electronic properties of $\mathrm{LaNiO}_{3}$ films. Phys. Rev. Appl. 2, 054004 (2014).

52. R. Scherwitzl, S. Gariglio, M. Gabay, P. Zubko, M. Gibert, and J.-M. Triscone: Metal-insulator transition in ultrathin $\mathrm{LaNiO}_{3}$ films. Phys. Rev. Lett. 106, 246403 (2011).

53. A.X. Gray, A. Janotti, J. Son, J.M. LeBeau, S. Ueda, Y. Yamashita, K. Kobayashi, A.M. Kaiser, R. Sutarto, 
H. Wadati, G.A. Sawatzky, C.G. Van de Walle, S. Stemmer, and C.S. Fadley: Insulating state of ultrathin epitaxial $\mathrm{LaNiO}_{3}$ thin films detected by hard x-ray photoemission. Phys. Rev. B 84, 075104 (2011)

54. J. Fowlie, M. Gibert, G. Tieri, A. Gloter, J. Íñiguez, A. Filippetti, S. Catalano, S. Gariglio, A. Schober, M. Guennou, J. Kreisel, O. Stéphan, and J.-M. Triscone: Conductivity and local structure of $\mathrm{LaNiO}_{3}$ thin films. $A d v$. Mater. 29, 1605197 (2017).

55. M. Golalikhani, Q. Lei, R.U. Chandrasena, L. Kasaei, H. Park, J. Bai, P. Orgiani, J. Ciston, G.E. Sterbinsky, D.A. Arena, P. Shafer, E. Arenholz, B.A. Davidson, A.J. Millis, A.X. Gray, and X.X. Xi: Nature of the metal-insulator transition in few-unit-cell-thick $\mathrm{LaNiO}_{3}$ films. Nat. Commun. 9, 2206 (2018).

56. D. Li, K. Lee, B.Y. Wang, M. Osada, S. Crossley, H.R. Lee, Y. Cui, Y. Hikita, and H.Y. Hwang: Superconductivity in an infinite-layer nickelate. Nature 572, 624 (2019).

57. K. Lee, B.H. Goodge, D. Li, M. Osada, B.Y. Wang, Y. Cui, L.F. Kourkoutis, and H.Y. Hwang: Aspects of the synthesis of thin film superconducting infinite-layer nickelates. APL Mater. 8, 041107 (2020).

58. M. Hepting, D. Li, C.J. Jia, H. Lu, E. Paris, Y. Tseng, X. Feng, M. Osada, E. Been, Y. Hikita, Y.-D. Chuang, Z. Hussain, K.J. Zhou, A. Nag, M. Garcia-Fernandez, M. Rossi, H.Y. Huang, D.J. Huang, Z.X. Shen, T. Schmitt, H.Y. Hwang, B. Moritz, J. Zaanen, T.P. Devereaux, and W.S. Lee: Electronic structure of the parent compound of superconducting infinitelayer nickelates. Nat. Mater. 19, 381 (2020).

59. S. Zeng, C.S. Tang, X. Yin, C. Li, Z. Huang, J. Hu, W. Liu, G.J. Omar, H. Jani, Z.S. Lim, K. Han, D. Wan, P. Yang, A.T.S. Wee, and A. Ariando: Phase diagram and superconducting dome of infinite-layer $\mathrm{Nd}_{1-\mathrm{x}} \mathrm{Sr}_{\mathrm{x}} \mathrm{NiO}_{2}$ thin films. arXiv:2004.11281 [cond-mat] (2020).

60. H. Hertz: Ueber einen Einfluss des ultravioletten Lichtes auf die electrische Entladung. Ann. Phys. 267, 983 (1887).

61. A. Einstein: Über einen die Erzeugung und Verwandlung des Lichtes betreffenden heuristischen Gesichtspunkt. Ann. Phys. 322, 132 (1905).

62. C. Nordling, E. Sokolowski, and K. Siegbahn: Precision method for obtaining absolute values of atomic binding energies. Phys. Rev. 105, 1676 (1957).

63. G.D. Mahan: Theory of photoemission in simple metals. Phys. Rev. B 2, 4334 (1970).

64. D. Briggs and J.T. Grant: Surface Analysis by Auger and X-Ray Photoelectron Spectroscopy (IM Publications, Chichester, West Sussex, U.K., 2003).

65. C.S. Fadley: (Hard X-ray Photoemission: An Overview and Future Perspective .In Hard X-Ray Photoelectron Spectroscopy
(HAXPES), J Woicik, ed. ((Springer International Publishing, Cham, 2016), pp. 1-34.

66. H. Matsuda, L. Tóth, and H. Daimon:

Variable-deceleration-ratio wide-acceptance-angle electrostatic lens for two-dimensional angular and energy analysis. Rev. Sci. Instrum. 89, 123105 (2018).

67. I. Niedermaier, C. Kolbeck, H.-P. Steinrück, and F. Maier: Dual analyzer system for surface analysis dedicated for angle-resolved photoelectron spectroscopy at liquid surfaces and interfaces. Rev. Sci. Instrum. 87, 045105 (2016).

68. S. Karkare, J. Feng, J. Maxson, and H.A. Padmore:

Development of a 3-D energy-momentum analyzer for meV-scale energy electrons. Rev. Sci. Instrum. 90, 053902 (2019).

69. M.P. Seah and W.A. Dench: Quantitative electron spectroscopy of surfaces: A standard data base for electron inelastic mean free paths in solids. Surf. Interface Anal. 1, 2 (1979).

70. D.R. Baer, K. Artyushkova, C. Richard Brundle, J.E. Castle, M.H. Engelhard, K.J. Gaskell, J.T. Grant, R.T. Haasch, M.R. Linford, C.J. Powell, A.G. Shard, P.M.A. Sherwood, and V.S. Smentkowski: Practical guides for $\mathrm{x}$-ray photoelectron spectroscopy: First steps in planning, conducting, and reporting XPS measurements. J. Vac. Sci. Technol. A 37, 031401 (2019).

71. C.J. Powell and S. Tanuma. "Inelastic Mean Free Paths, Mean Escape Depths, Information Depths, and Effective Attenuation Lengths for Hard X-ray Photoelectron Spectroscopy" In Hard $X$-Ray Photoelectron Spectroscopy (HAXPES), J. Woicik, ed. (Springer International Publishing, Cham, 2016); pp. 111-140.

72. C.S. Fadley and D.A. Shirley: Multiplet splitting of metal-atom electron binding energies. Phys. Rev. A 2, 1109 (1970).

73. E.S. Ilton, J.E. Post, P.J. Heaney, F.T. Ling, and S.N. Kerisit: XPS determination of Mn oxidation states in Mn (hydr)oxides. Appl. Surf. Sci. 366, 475 (2016).

74. L. Qiao, H.Y. Xiao, S.M. Heald, M.E. Bowden, T. Varga, G.J. Exarhos, M.D. Biegalski, I.N. Ivanov, W.J. Weber, T.C. Droubay, and S.A. Chambers: The impact of crystal symmetry on the electronic structure and functional properties of complex lanthanum chromium oxides. J. Mater. Chem. C 1, 4527 (2013).

75. M.A. Brisk and A.D. Baker: Shake-up satellites in X-ray photoelectron spectroscopy. J. Electron Spectrosc. Relat. Phenom. 7, 197 (1975).

76. K.S. Kim: X-ray-photoelectron spectroscopic studies of the electronic structure of CoO. Phys. Rev. B 11, 2177 (1975).

77. K.S. Kim and N. Winograd: Charge transfer shake-up satellites in X-ray photoelectron spectra of cations and anions of $\mathrm{SrTiO}_{3}$, $\mathrm{TiO}_{2}$ and $\mathrm{Sc}_{2} \mathrm{O}_{3}$. Chem. Phys. Lett. 31, 312 (1975).

78. C.S. Fadley, S.B.M. Hagstrom, M.P. Klein, and D.A. Shirley: Chemical effects on core-electron binding energies in iodine and europium. J. Chem. Phys. 48, 3779 (1968). 
79. S.A. Chambers, T.C. Droubay, C. Capan, and G.Y. Sun: Unintentional $\mathrm{F}$ doping of $\mathrm{SrTiO}_{3}(001)$ etched in HF acid-structure and electronic properties. Surf. Sci. 606, 554 (2012).

80. M. Kawasaki, K. Takahashi, T. Maeda, R. Tsuchiya, M. Shinohara, O. Ishiyama, T. Yonezawa, M. Yoshimoto, and H. Koinuma: Atomic control of the $\mathrm{SrTiO}_{3}$ crystal surface. Science 266, 1540 (1994).

81. J.H. Lee, G. Luo, I.C. Tung, S.H. Chang, Z. Luo, M. Malshe, M. Gadre, A. Bhattacharya, S.M. Nakhmanson, J.A. Eastman, H. Hong, J. Jellinek, D. Morgan, D.D. Fong, and

J.W. Freeland: Dynamic layer rearrangement during growth of layered oxide films by molecular beam epitaxy. Nat. Mater. 13, 879 (2014).

82. Y.F. Nie, Y. Zhu, C.-H. Lee, L.F. Kourkoutis, J.A. Mundy, J. Junquera, P. Ghosez, D.J. Baek, S. Sung, X.X. Xi, K.M. Shen, D.A. Muller, and D.G. Schlom: Atomically precise interfaces from non-stoichiometric deposition. Nat. Commun. 54530 (2014).

83. S. Thapa, S.R. Provence, W. Jin, J. Lapano, J.T. Sadowski, M. Brahlek, and R.B. Comes: Correlating surface stoichiometry and termination in $\mathrm{SrTiO}_{3}$ films grown by hybrid molecular beam epitaxy. arXiv:2004.00069 (2020).

84. A. Berlich, H. Strauss, C. Langheinrich, A. Chassé, and H. Morgner: Surface termination of $\mathrm{BaTiO}_{3}$ (001) single crystals, A combined electron spectroscopic and theoretical study. Surf. Sci. 605, 158 (2011).

85. G. Singh-Bhalla, P.B. Rossen, G.K. Pálsson, M. Mecklenburg, T. Orvis, S. Das, Y.-L. Tang, J.S. Suresha, D. Yi, A. Dasgupta, D. Doenning, V.G. Ruiz, A.K. Yadav, M. Trassin, J.T. Heron, C.S. Fadley, R. Pentcheva, J. Ravichandran, and R. Ramesh: Unexpected termination switching and polarity compensation in $\mathrm{LaAlO}_{3} / \mathrm{SrTiO}_{3}$ heterostructures. Phys. Rev. Mater. 2, 112001 (2018).

86. K.A. Stoerzinger, R. Comes, S.R. Spurgeon, S. Thevuthasan, K. Ihm, E.J. Crumlin, and S.A. Chambers: Influence of $\mathrm{LaFeO}_{3}$ surface termination on water reactivity. J. Phys. Chem. Lett. 8, 1038 (2017).

87. D. Halwidl, W. Mayr-Schmölzer, D. Fobes, J. Peng, Z. Mao, M. Schmid, F. Mittendorfer, J. Redinger, and U. Diebold: Ordered hydroxyls on $\mathrm{Ca}_{3} \mathrm{Ru}_{2} \mathrm{O}_{7}$ (001). Nat. Commun. 8, 23 (2017).

88. D. Halwidl, B. Stöger, W. Mayr-Schmölzer, J. Pavelec, D. Fobes, J. Peng, Z. Mao, G.S. Parkinson, M. Schmid, F. Mittendorfer, J. Redinger, and U. Diebold: Adsorption of water at the SrO surface of ruthenates. Nat. Mater. 15, 450 (2016).

89. L. Qiao, T.C. Droubay, T. Varga, M.E. Bowden, V. Shutthanandan, Z. Zhu, T.C. Kaspar, and S.A. Chambers: Epitaxial growth, structure, and intermixing at the $\mathrm{LaAlO}_{3} /$
$\mathrm{SrTiO}_{3}$ interface as the film stoichiometry is varied. Phys. Rev. B 83, 085408 (2011).

90. G. Salvinelli, G. Drera, A. Giampietri, and L. Sangaletti: Layer-resolved cation diffusion and stoichiometry at the $\mathrm{LaAlO}_{3}$ / $\mathrm{SrTiO}_{3}$ heterointerface probed by X-ray photoemission experiments and site occupancy modeling. ACS Appl. Mater. Interfaces 7, 25648 (2015).

91. M. Sing, G. Berner, K. Goß, A. Müller, A. Ruff, A. Wetscherek, S. Thiel, J. Mannhart, S.A. Pauli, C.W. Schneider, P.R. Willmott, M. Gorgoi, F. Schäfers, and R. Claessen: Profiling the interface electron gas of $\mathrm{LaAlO}_{3} / \mathrm{SrTiO}_{3}$ heterostructures with hard X-ray photoelectron spectroscopy. Phys. Rev. Lett. 102, 176805 (2009).

92. Y.Z. Chen, N. Bovet, F. Trier, D.V. Christensen, F.M. Qu, N.H. Andersen, T. Kasama, W. Zhang, R. Giraud, J. Dufouleur, T.S. Jespersen, J.R. Sun, A. Smith, J. Nygård, L. Lu, B. Büchner, B.G. Shen, S. Linderoth, and N. Pryds: A high-mobility two-dimensional electron gas at the spinel/ perovskite interface of $\gamma-\mathrm{Al}_{2} \mathrm{O}_{3} / \mathrm{SrTiO}_{3}$. Nat. Commun. 4, 1371 (2013).

93. M. Saghayezhian, Z. Wang, H. Guo, Y. Zhu, E.W. Plummer, and J. Zhang: Manipulating the polar mismatch at the $\mathrm{LaNiO}_{3} /$ $\mathrm{SrTiO}_{3}$ (111) interface. Phys. Rev. B 95, 165434 (2017).

94. R.B. Comes, S.R. Spurgeon, D.M. Kepaptsoglou, M.H. Engelhard, D.E. Perea, T.C. Kaspar, Q.M. Ramasse, P.V. Sushko, and S.A. Chambers: Probing the origin of interfacial carriers in $\mathrm{SrTiO}_{3}-\mathrm{LaCrO}_{3}$ superlattices. Chem. Mater. 29, 1147 (2017).

95. E.A. Kraut, R.W. Grant, J.R. Waldrop, and S.P. Kowalczyk: Precise determination of the valence-band edge in X-ray photoemission spectra: Application to measurement of semiconductor interface potentials. Phys. Rev. Lett. 44, 1620 (1980).

96. S.A. Chambers, Y. Du, R.B. Comes, S.R. Spurgeon, and P.V. Sushko: The effects of core-level broadening in determining band alignment at the epitaxial $\mathrm{SrTiO}_{3}(001) / p-\mathrm{Ge}(001)$ heterojunction. Appl. Phys. Lett. 110, 082104 (2017).

97. S.A. Chambers, T. Droubay, T.C. Kaspar, and M. Gutowski: Experimental determination of valence band maxima for $\mathrm{SrTiO}_{3}$, $\mathrm{TiO}_{2}$, and $\mathrm{SrO}$ and the associated valence band offsets with $\mathrm{Si}$ (001). J. Vac. Sci. Technol. B 22, 2205 (2004).

98. L. Qiao, T.C. Droubay, V. Shutthanandan, Z. Zhu, P.V. Sushko, and S.A. Chambers: Thermodynamic instability at the stoichiometric $\mathrm{LaAlO}_{3} / \mathrm{SrTiO}_{3}(001)$ interface. J. Phys.: Condens. Matter 22, 312201 (2010).

99. G. Drera, G. Salvinelli, A. Brinkman, M. Huijben, G. Koster, H. Hilgenkamp, G. Rijnders, D. Visentin, and L. Sangaletti: Band offsets and density of $\mathrm{Ti}^{3+}$ states probed by $\mathrm{x}$-ray photoemission on $\mathrm{LaAlO}_{3} / \mathrm{SrTiO}_{3}$ heterointerfaces and their $\mathrm{LaAlO}_{3}$ and $\mathrm{SrTiO}_{3}$ bulk precursors. Phys. Rev. B 87, 075435 (2013). 
100. A. Giampietri, G. Drera, and L. Sangaletti: Band alignment at heteroepitaxial perovskite oxide interfaces. Experiments, methods, and perspectives. Adv. Mater. Interfaces 4, 1700144 (2017).

101. R.B. Comes, P. Xu, B. Jalan, and S.A. Chambers: Band alignment of epitaxial $\mathrm{SrTiO}_{3}$ thin films with $\left(\mathrm{LaAlO}_{3}\right)_{0.3}-\left(\mathrm{Sr}_{2} \mathrm{AlTaO}_{6}\right)_{0.7}(001)$. Appl. Phys. Lett. 107, 131601 (2015).

102. R. Schafranek, J.D. Baniecki, M. Ishii, Y. Kotaka, K. Yamanka, and K. Kurihara: Band offsets at the epitaxial $\mathrm{SrTiO}_{3} / \mathrm{SrZrO}_{3}$ (001) heterojunction. J. Phys. D: Appl. Phys. 45, 055303 (2012).

103. S.A. Chambers, T.C. Kaspar, A. Prakash, G. Haugstad, and B. Jalan: Band alignment at epitaxial $\mathrm{BaSnO}_{3} / \mathrm{SrTiO}_{3}(001)$ and $\mathrm{BaSnO}_{3} / \mathrm{LaAlO}_{3}(001)$ heterojunctions. Appl. Phys. Lett. 108, 152104 (2016)

104. Z. Zhong and P. Hansmann: Band alignment and charge transfer in complex oxide interfaces. Phys. Rev. X 7, 011023 (2017).

105. M. Nakamura, F. Kagawa, T. Tanigaki, H.S. Park, T. Matsuda, D. Shindo, Y. Tokura, and M. Kawasaki: Spontaneous polarization and bulk photovoltaic effect driven by polar discontinuity in $\mathrm{LaFeO}_{3} / \mathrm{SrTiO}_{3}$ heterojunctions. Phys. Rev. Lett. 116, 156801 (2016).

106. K. Nakamura, H. Mashiko, K. Yoshimatsu, and A. Ohtomo: Impact of built-in potential across $\mathrm{LaFeO}_{3} / \mathrm{SrTiO}_{3}$ heterojunctions on photocatalytic activity. Appl. Phys. Lett. 108, 211605 (2016).

107. R. Comes and S. Chambers: Interface structure, band alignment, and built-in potentials at $\mathrm{LaFeO}_{3} / \mathrm{n}-\mathrm{SrTiO}_{3}$ heterojunctions. Phys. Rev. Lett. 117, 226802 (2016).

108. P. Xu, W. Han, P.M. Rice, J. Jeong, M.G. Samant, K. Mohseni, H.L. Meyerheim, S. Ostanin, I.V. Maznichenko, I. Mertig,

E.K.U. Gross, A. Ernst, and S.S.P. Parkin: Reversible formation of $2 \mathrm{D}$ electron gas at the $\mathrm{LaFeO}_{3} / \mathrm{SrTiO}_{3}$ interface via control of oxygen vacancies. Adv. Mater. 29, 1604447 (2017).

109. S.R. Spurgeon, P.V. Sushko, S.A. Chambers, and R.B. Comes: Dynamic interface rearrangement in $\mathrm{LaFeO}_{3} / \mathrm{n}-\mathrm{SrTiO}_{3}$ heterojunctions. Phys. Rev. Mater. 1, 063401 (2017).

110. J.E. Kleibeuker, Z. Zhong, H. Nishikawa, J. Gabel, A. Müller, F. Pfaff, M. Sing, K. Held, R. Claessen, G. Koster, and G. Rijnders: Electronic reconstruction at the isopolar $\mathrm{LaTiO}_{3} /$ $\mathrm{LaFeO}_{3}$ interface: An X-ray photoemission and densityfunctional theory study. Phys. Rev. Lett. 113, 237402 (2014).

111. G. Araizi-Kanoutas, J. Geessinck, N. Gauquelin, S. Smit, X.H. Verbeek, S.K. Mishra, P. Bencok, C. Schlueter, T.-L. Lee, D. Krishnan, J. Fatermans, J. Verbeeck, G. Rijnders, G. Koster, and M.S. Golden: Co valence transformation in isopolar $\mathrm{LaCoO}_{3} / \mathrm{LaTiO}_{3}$ perovskite heterostructures via interfacial engineering. Phys. Rev. Mater. 4, 026001 (2020).

112. L. Qiao and X. Bi: Direct observation of $\mathrm{Ni}^{3+}$ and $\mathrm{Ni}^{2+}$ in correlated $\mathrm{LaNiO}_{3-\delta}$ films. EPL 93, 57002 (2011).

113. R. Reininger, J.C. Woicik, S.L. Hulbert, and D.A. Fischer: NIST NSLS-II spectroscopy beamline optical plan for soft and tender X-ray spectroscopy and microscopy ( $100 \mathrm{eV}$ to $7.5 \mathrm{keV})$.

Nucl. Instrum. Methods Phys. Res. A 649, 49 (2011).

114. J.-P. Rueff, J.E. Rault, J.M. Ablett, Y. Utsumi, and D. Céolin: HAXPES for materials science at the GALAXIES beamline. Synchrotron Radiat. News 31, 4 (2018).

115. C. Schlueter, A. Gloskovskii, K. Ederer, I. Schostak, S. Piec, I. Sarkar, Y. Matveyev, P. Lömker, M. Sing, R. Claessen, C. Wiemann, C.M. Schneider, K. Medjanik, G. Schönhense, P. Amann, A. Nilsson, and W. Drube: The new dedicated HAXPES beamline P22 at PETRAIII. AIP Conf. Proc. 2054, 040010 (2019).

116. S. Yasuno, H. Oji, T. Koganezawa, and T. Watanabe: Hard $\mathrm{X}$-ray photoelectron spectroscopy equipment developed at beamline BL46XU of SPring- 8 for industrial researches. AIP Conf. Proc. 1741, 030020 (2016).

117. J. Gabel, M. Zapf, P. Scheiderer, P. Schütz, L. Dudy, M. Stübinger, C. Schlueter, T.-L. Lee, M. Sing, and R. Claessen: Disentangling specific versus generic doping mechanisms in oxide heterointerfaces. Phys. Rev. B 95, 195109 (2017).

118. C.S. Fadley and S. Nemšák: Some future perspectives in soft- and hard- X-ray photoemission. J. Electron Spectrosc. Relat. Phenom. 195, 409 (2014).

119. S.A. Chambers. "Probing Perovskite Interfaces and Superlattices with X-ray Photoemission Spectroscopy" In Hard X-Ray Photoelectron Spectroscopy (HAXPES), J. Woicik, ed. (Springer International Publishing, Cham, 2016); pp. 341-380.

120. M. Sing, and R. Claessen. "Photoelectron Spectroscopy of Transition-Metal Oxide Interfaces" In Spectroscopy of Complex Oxide Interfaces: Photoemission and Related Spectroscopies, C. Cancellieri, and V.N. Strocov, eds. (Springer International Publishing, Cham, 2018); pp. 87-105.

121. S. Gariglio and C. Cancellieri. "The $\mathrm{LaAlO} 3$ / SrTiO 3 Interface: The Origin of the 2D Electron Liquid and the Fabrication" In Spectroscopy of Complex Oxide Interfaces: Photoemission and Related Spectroscopies, C. Cancellieri, and V.N. Strocov, eds. (Springer International Publishing, Cham, 2018); pp. 17-35.

122. H. Paik, Z. Chen, E. Lochocki, A. Seidner H, A. Verma, N. Tanen, J. Park, M. Uchida, S. Shang, B.-C. Zhou, M. Brützam, R. Uecker, Z.-K. Liu, D. Jena, K.M. Shen, D.A. Muller, and D.G. Schlom: Adsorption-controlled growth of La-doped $\mathrm{BaSnO}_{3}$ by molecular-beam epitaxy. APL Mater. 5, 116107 (2017).

123. A. Prakash, P. Xu, A. Faghaninia, S. Shukla, J.W. Ager Iii, C.S. Lo, and B. Jalan: Wide bandgap $\mathrm{BaSnO}_{3}$ films with room temperature conductivity exceeding $10^{4} \mathrm{~S} \mathrm{~cm}^{-1}$. Nat. Commun. 8 , 15167 (2017).

124. S. Raghavan, T. Schumann, H. Kim, J.Y. Zhang, T.A. Cain, and S. Stemmer: High-mobility $\mathrm{BaSnO}_{3}$ grown by oxide molecular beam epitaxy. APL Mater. 4, 016106 (2016). 
125. S. Sallis, D.O. Scanlon, S.C. Chae, N.F. Quackenbush, D.A. Fischer, J.C. Woicik, J.-H. Guo, S.W. Cheong, and L.F.J. Piper: La-doped $\mathrm{BaSnO}_{3}-$ Degenerate perovskite transparent conducting oxide: Evidence from synchrotron $\mathrm{x}$-ray spectroscopy. Appl. Phys. Lett. 103, 042105 (2013).

126. Z. Lebens-Higgins, D.O. Scanlon, H. Paik, S. Sallis, Y. Nie, M. Uchida, N.F. Quackenbush, M.J. Wahila, G.E. Sterbinsky, D.A. Arena, J.C. Woicik, D.G. Schlom, and L.F.J. Piper: Direct observation of electrostatically driven band gap renormalization in a degenerate perovskite transparent conducting oxide. Phys. Rev. Lett. 116, 027602 (2016).

127. C. Park, U. Kim, C.J. Ju, J.S. Park, Y.M. Kim, and K. Char: High mobility field effect transistor based on $\mathrm{BaSnO}_{3}$ with $\mathrm{Al}_{2} \mathrm{O}_{3}$ gate oxide. Appl. Phys. Lett. 105, 203503 (2014).

128. J. Park, H. Paik, K. Nomoto, K. Lee, B.-E. Park, B. Grisafe, L.-C. Wang, S. Salahuddin, S. Datta, Y. Kim, D. Jena, H.G. Xing, and D.G. Schlom: Fully transparent field-effect transistor with high drain current and on-off ratio. APL Mater. 8, 011110 (2020).

129. K. Krishnaswamy, L. Bjaalie, B. Himmetoglu, A. Janotti, L. Gordon, and C.G. Van de Walle: $\mathrm{BaSnO}_{3}$ as a channel material in perovskite oxide heterostructures. Appl. Phys. Lett. 108, 083501 (2016).

130. L. Weston, L. Bjaalie, K. Krishnaswamy, and C.G. Van de Walle: Origins of $n$-type doping difficulties in perovskite stannates. Phys. Rev. B 97, 054112 (2018).

131. J. Zhang, S. Han, W. Luo, S. Xiang, J. Zou, F.E. Oropeza, M. Gu, and K.H.L. Zhang: Interface energy band alignment at the all-transparent $\mathrm{p}$-n heterojunction based on $\mathrm{NiO}$ and $\mathrm{BaSnO}_{3}$. Appl. Phys. Lett. 112, 171605 (2018).

132. A. Prakash, N.F. Quackenbush, H. Yun, J. Held, T. Wang, T. Truttmann, J.M. Ablett, C. Weiland, T.-L. Lee, J.C. Woicik, K.A. Mkhoyan, and B. Jalan: Separating electrons and donors in $\mathrm{BaSnO}_{3}$ via band engineering. Nano Lett. 19, 8920 (2019).

133. A.X. Gray: Future directions in standing-wave photoemission. J. Electron Spectrosc. Relat. Phenom. 195, 399 (2014).

134. A.X. Gray, S. Nemšák, and C.S. Fadley: Combining hard and soft X-ray photoemission with standing-wave excitation, resonant excitation, and angular resolution. Synchrotron Radiat. News 31, 42 (2018).

135. S. Nemšák, A.X. Gray, and C.S. Fadley. "Standing-Wave and Resonant Soft- and Hard-X-ray Photoelectron Spectroscopy of Oxide Interfaces" In Spectroscopy of Complex Oxide Interfaces: Photoemission and Related Spectroscopies, C. Cancellieri, and V.N. Strocov, eds. (Springer International Publishing, Cham, 2018); pp. 153-179.

136. S.-C. Lin, C.-T. Kuo, R.B. Comes, J.E. Rault, J.-P. Rueff, S. Nemšák, A. Taleb, J.B. Kortright, J. Meyer-Ilse, E. Gullikson,
P.V. Sushko, S.R. Spurgeon, M. Gehlmann, M.E. Bowden,

L. Plucinski, S.A. Chambers, and C.S. Fadley: Interface properties and built-in potential profile of a $\mathrm{LaCrO}_{3} / \mathrm{SrTiO}_{3}$ superlattice determined by standing-wave excited photoemission spectroscopy. Phys. Rev. B 98, 165124 (2018).

137. R.B. Comes, S.R. Spurgeon, S.M. Heald, D.M. Kepaptsoglou,

L. Jones, P.V. Ong, M.E. Bowden, Q.M. Ramasse, P.V. Sushko, and S.A. Chambers: Interface-induced polarization in $\mathrm{SrTiO}_{3}-\mathrm{LaCrO}_{3}$ Superlattices. Adv. Mater. Interfaces 3, 201500779 (2016).

138. A.M. Kaiser, A.X. Gray, G. Conti, J. Son, A. Greer, A. Perona, A. Rattanachata, A.Y. Saw, A. Bostwick, S. Yang, S.-H. Yang, E.M. Gullikson, J.B. Kortright, S. Stemmer, and C.S. Fadley: Suppression of near-Fermi level electronic states at the interface in a $\mathrm{LaNiO}_{3} / \mathrm{SrTiO}_{3}$ superlattice. Phys. Rev. Lett. 107, 116402 (2011).

139. R.U. Chandrasena, C.L. Flint, W. Yang, A. Arab, S. Nemšák, M. Gehlmann, V.B. Özdöl, F. Bisti, K.D. Wijesekara, J. Meyer-Ilse, E. Gullikson, E. Arenholz, J. Ciston, C.M. Schneider, V.N. Strocov, Y. Suzuki, and A.X. Gray: Depth-resolved charge reconstruction at the $\mathrm{LaNiO}_{3} / \mathrm{CaMnO}_{3}$ interface. Phys. Rev. B 98, 155103 (2018).

140. A.X. Gray, C. Papp, B. Balke, S.-H. Yang, M. Huijben, E. Rotenberg, A. Bostwick, S. Ueda, Y. Yamashita, K. Kobayashi, E.M. Gullikson, J.B. Kortright, F.M.F. de Groot, G. Rijnders, D.H.A. Blank, R. Ramesh, and C.S. Fadley: Interface properties of magnetic tunnel junction $\mathrm{La}_{0.7} \mathrm{Sr}_{0.3} \mathrm{MnO}_{3} /$ $\mathrm{SrTiO}_{3}$ superlattices studied by standing-wave excited photoemission spectroscopy. Phys. Rev. B 82, 205116 (2010).

141. S. Nemšák, G. Conti, A.X. Gray, G.K. Palsson, C. Conlon, D. Eiteneer, A. Keqi, A. Rattanachata, A.Y. Saw, A. Bostwick, L. Moreschini, E. Rotenberg, V.N. Strocov, M. Kobayashi, T. Schmitt, W. Stolte, S. Ueda, K. Kobayashi, A. Gloskovskii, W. Drube, C.A. Jackson, P. Moetakef, A. Janotti, L. Bjaalie, B. Himmetoglu, C.G. Van de Walle, S. Borek, J. Minar, J. Braun, H. Ebert, L. Plucinski, J.B. Kortright, C.M. Schneider, L. Balents, F.M.F. de Groot, S. Stemmer, and C.S. Fadley: Energetic, spatial, and momentum character of the electronic structure at a buried interface: The two-dimensional electron gas between two metal oxides. Phys. Rev. B 93, 245103 (2016).

142. A.X. Gray, J. Minár, L. Plucinski, M. Huijben, A. Bostwick, E. Rotenberg, S.-H. Yang, J. Braun, A. Winkelmann, G. Conti, D. Eiteneer, A. Rattanachata, A.A. Greer, J. Ciston, C. Ophus, G. Rijnders, D.H.A. Blank, D. Doennig, R. Pentcheva, J.B. Kortright, C.M. Schneider, H. Ebert, and C.S. Fadley: Momentum-resolved electronic structure at a buried interface from soft X-ray standing-wave angle-resolved photoemission. EPL 104, 17004 (2013). 
143. S. Döring, F. Schönbohm, U. Berges, R. Schreiber,

D.E. Bürgler, C.M. Schneider, M. Gorgoi, F. Schäfers, C. Papp,

B. Balke, C.S. Fadley, and C. Westphal: Hard X-ray photoemission using standing-wave excitation applied to the $\mathrm{MgO} / \mathrm{Fe}$ interface. Phys. Rev. B 83, 165444 (2011).

144. S. Nemšák, A. Shavorskiy, O. Karslioglu, I. Zegkinoglou,
A. Rattanachata, C.S. Conlon, A. Keqi, P.K. Greene,
E.C. Burks, F. Salmassi, E.M. Gullikson, S.-H. Yang, K. Liu,
H. Bluhm, and C.S. Fadley: Concentration and chemical-state profiles at heterogeneous interfaces with sub-nm accuracy from standing-wave ambient-pressure photoemission. Nat.
Commun. 5, 5441 (2014).

145. E. Gullikson: X-Ray Interactions with Matter (Lawrence Berkeley National Laboratory, Berkeley, CA USA, 2010).

146. B.L. Henke, E.M. Gullikson, and J.C. Davis: X-ray interactions: Photoabsorption, scattering, transmission, and reflection at $\mathrm{E}=50$ 30,000 eV, Z=1-92. At. Data Nucl. Data Tables 54, 181 (1993).
147. R.M. Palomino, E. Stavitski, I. Waluyo, Y.K. Chen-Wiegart, M. Abeykoon, J.T. Sadowski, J.A. Rodriguez, A.I. Frenkel, and S.D. Senanayake: New in-situ and operando facilities for catalysis science at NSLS-II: The deployment of real-time, chemical, and structure-sensitive X-ray probes. Synchrotron Radiat. News 30, 30 (2017).

148. G. Eres, C.M. Rouleau, Q. Lu, Z. Zhang, E. Benda, H.N. Lee, J.Z. Tischler, and D.D. Fong: Experimental setup combining in situ hard X-ray photoelectron spectroscopy and real-time surface $\mathrm{X}$-ray diffraction for characterizing atomic and electronic structure evolution during complex oxide heterostructure growth. Rev. Sci. Instrum. 90, 093902 (2019).

149. A. Regoutz, M. Mascheck, T. Wiell, S.K. Eriksson,

C. Liljenberg, K. Tetzner, B.A.D. Williamson, D.O. Scanlon, and P. Palmgren: A novel laboratory-based hard X-ray photoelectron spectroscopy system. Rev. Sci. Instrum. 89, 073105 (2018). 